J. Math. Soc. Japarı

Vol. 31, No. 3, 1979

\title{
Automorphic forms and the periods of abelian varieties
}

\author{
By Goro SHIMURA*
}

(Received July 11, 1978)

There are three interrelated topics to be treated in this paper:

I. Monomial relations between the periods of abelian varieties with complex multiplication;

II. The derivatives of automorphic forms of arithmetic type;

III. The non-vanishing of the first cohomology group of a discrete subgroup of $S U(n, 1)$.

To describe our results, let $A$ be an abelian variety of dimension $g$ defined over $\overline{\boldsymbol{Q}}$, whose endomorphism-algebra contains a totally imaginary quadratic extension $K$ of a totally real algebraic number field $F$ such that $[F: \boldsymbol{Q}]=g$. Throughout the paper, we denote by $\overline{\boldsymbol{Q}}$ the algebraic closure of the rational number field $\boldsymbol{Q}$ embedded in the complex number field $\boldsymbol{C}$. Let $\Phi$ be the representation of $K$ on the space of holomorphic 1-forms on $A$. Then $\Phi$ consists of $g$ injections $\tau$ of $K$ into $\boldsymbol{C}$, and for each $\tau$, there is a $\overline{\boldsymbol{Q}}$-rational 1 -form $\omega_{\tau}$ on $A$ on which an element $x$ of $K$ acts as a scalar $x^{\tau}$. As shown in [13], there is a constant $p(\tau, \Phi)$ such that

$$
\int_{c} \omega_{\tau} \sim \pi \cdot p(\tau, \Phi) \quad \text { for all } 1 \text {-cycles } c \text { on } A \text {. }
$$

Here and henceforth, we write $a \sim b$ for two complex numbers $a$ and $b$ if $a / b \in \overline{\boldsymbol{Q}}$. The first principal aim of this paper is to prove monomial relations between $p(\tau, \Phi)$ for various $\Phi$ with the same $K$, as well as relations between such "periods" for a given $K$ and those for an extension of $K$. The constant $p(\tau, \Phi)$ can be obtained from a Hilbert modular function $f$ with respect to a congruence subgroup of $G L_{2}(F)$ as follows. Take the variable $z=\left(z_{1}, \cdots, z_{g}\right)$ on the product $\mathscr{S}_{1}^{g}$ of $g$ copies of the upper half plane $\mathscr{S}_{1}$. Let $\Phi=\sum_{\nu=1}^{g} \tau_{\nu}$ and $w_{0}=\left(w^{\tau_{1}}, \cdots, w^{\tau} g\right)$ with an element $w$ of $K$ such that $\operatorname{Im}\left(w^{\tau_{\nu}}\right)>0$ for all $\nu$. Then

$$
\left(\partial f / \partial z_{\nu}\right)\left(w_{0}\right) \sim \pi \cdot p\left(\tau_{\nu}, \Phi\right)^{2} \quad(\nu=1, \cdots, g),
$$

if $f$ is $\overline{\mathbf{Q}}$-rational, i. e., if $f$ is the quotient of two Hilbert modular forms with

* Supported by NSF Grant MCS76-11376. 
Fourier coefficients in $\overline{\boldsymbol{Q}}$ (see [13]). Now we can speak of $\overline{\boldsymbol{Q}}$-rational automorphic functions on $\mathscr{S}_{1}^{g}$ for a more general type of algebraic groups of which $G L_{2}(F)$ is a special case. The generalization of $(0.2)$ to such functions will be our second main subject.

As the third topic, which is a sort of by-product, we show the existence of a discrete subgroup $\Gamma$ of $S U(n, 1)$ such that $H^{1}\left(\Gamma \backslash \mathfrak{D}_{n}, \boldsymbol{R}\right) \neq\{0\}$, where

$$
\mathfrak{D}_{n}=\left\{\left.\left(z_{1}, \cdots, z_{n}\right) \in \boldsymbol{C}^{n}\left|\sum_{k=1}^{n}\right| z_{k}\right|^{2}<1\right\} .
$$

Such a non-vanishing was first proved by Kazhdan in [3]. Subsequently Wallach obtained more general results for $S U(p, q)$ in the case of compact quotient [1, Ch. VIII]. ${ }^{1)}$

Our results will be obtained by considering a family of abelian varieties of dimension $m g$ with the above $K$ as a subalgebra of the endomorphismalgebra. This is parametrized by the points of a symmetric domain $\mathscr{D}$ of which $\mathscr{D}_{n}$ is a special case. We embed $\mathfrak{D}$ into the Siegel upper half space $\mathfrak{H}_{m g}$ of degree $m g$, and pull back a certain modular form on $\mathfrak{S}_{m g}$ to $\mathfrak{D}$, which gives rise to a non-vanishing holomorphic 1 -form on $\Gamma \backslash \mathfrak{D}_{m-1}$ when $\mathfrak{D}=\mathfrak{D}_{m-1}$. The study of the first two topics are made by analysing the pull-back of another type of (meromorphic) modular form at $C M$-points. This combined with our previous results concerning 1 -forms on $A$ in [13] yields the main theorems on the monomial relations of $p(\tau, \Phi)$ as well as those on the generalization of $(0.2)$,

Obviously our investigation of the second topic admits of a further extension to the algebraic groups of a more general type; even for the groups in the present paper, no effort is expended to make the sharpest statement. The author believes, however, that our theorems and propositions are given to such an extent as to explain the basic ideas and indicate what their generalizations or analogues in other cases should be.

\section{The main theorems on monomial relations between the periods.}

We let an isomorphism $\sigma$ of a field act on the right; thus $x^{\sigma}$ denotes the image under $\sigma$ of an element $x$ of the field. Throughout the paper, we denote by $\rho$ the complex conjugation. By a $C M$-field, we understand an algebraic number field $K$ of finite degree with an automorphism $\sigma$ of order 2 such that $\sigma \tau=\tau \rho$ for every injection $\tau$ of $K$ into $C$. Such a $\sigma$ is obviously unique for $K$; therefore we use the same letter $\rho$ for $\sigma$; we also denote by $K_{0}$ the fixed subfield of $K$ by $\rho$. Then $K_{0}$ is totally real and $K$ is a totally

1) The reader who is interested only in this topic may dispense with $\S \S 2,5,6$, and 7. 
imaginary quadratic extension of $K_{0}$. Conversely, such an extension is a $C M$-field.

Given an algebraic number field $K$ of finite degree, we denote by $I_{K}$ (resp. $I_{K}(\boldsymbol{Q})$ ) the module of all formal linear combinations $\Phi=\sum_{\tau} c_{\tau} \tau$ with $c_{\tau} \in \boldsymbol{Z}$ (resp. $c_{\tau} \in \boldsymbol{Q}$ ) of the injections $\tau$ of $K$ into $\boldsymbol{C}$. If $c_{\tau} \geqq 0$ for all $\tau$, $\Phi$ may be considered an equivalence class of representations of $K$ by complex matrices; we then put $\operatorname{tr} \Phi(a)=\sum_{\tau} c_{\tau} a^{\tau}$ for $a \in K$. We call $(K, \Phi)$ a $C M$-type if $K$ is a $C M$-field and the sum of $\Phi$ and its complex conjugate $\Phi \rho$ is the class of regular representations of $K$ over $\boldsymbol{Q}$. We shall also say that $\Phi$ is a $C M$-type of $K$.

Let $A$ be an abelian variety defined over $C$, and $c$ an injection of $K$ into $\operatorname{End}(A) \otimes \boldsymbol{Q}$. We say that $(A, \iota)$ is of type $(K, \Phi)$ if the representation of $K$ through $c$ on the space of holomorphic 1-forms on $A$ belongs to $\Phi$. Suppose $(K, \Phi)$ is a $C M$-type and $(A, \iota)$ is of type $(K, \Phi)$ and defined over $\overline{\boldsymbol{Q}}$. Let $[K: \boldsymbol{Q}]=2 g$ and $\Phi=\sum_{=1}^{g} \tau_{\nu}$. For each $\nu$, there is a $\overline{\boldsymbol{Q}}$-rational holomorphic 1 -form $\omega_{\nu}$ on $A$ satisfying $\omega_{\nu} \iota c(a)=a^{\tau} \nu \omega_{\nu}$ for all $a \in K$ such that $\iota(a) \in \operatorname{End}(A)$; $\omega_{\nu}$ is unique up to algebraic factors. In [13, Remark 3.4], we have proved:

Proposition 1.1. There exists a non-zero complex number $p\left(\tau_{\nu}, \Phi\right)$ depending only on $K, \Phi$, and $\tau_{\nu}$ such that $\int_{c} \omega_{\nu} \sim \pi \cdot p\left(\tau_{\nu}, \Phi\right)$ for all 1-cycles $c$ on $A$.

The constant $p\left(\tau_{\nu}, \Phi\right)$ is determined up to algebraic factors. If the multiplicity of $\tau$ in $\Phi$ is 0 , (i. e., if $\tau \notin\left\{\tau_{1}, \cdots, \tau_{g}\right\}$,) we simply define $p(\tau, \Phi)$ to be 1 . Now our first main result, in a (seemingly) weaker form, can be stated as

THEOREM 1.2. Let $\Phi_{1}, \cdots, \Phi_{m}$ be CM-types of a CM-field $K$, and $\tau$ an injection of $K$ into $\boldsymbol{C}$. Then the number

$$
\Pi_{j=1}^{m}\left[p\left(\tau, \Phi_{j}\right) / p\left(\tau \rho, \Phi_{j}\right)\right],
$$

up to algebraic factors, depends only on $\tau$ and $\Phi_{1}+\cdots+\Phi_{m}$. Moreover, let $(L, \Psi)$ be a CM-type such that $K \subset L$ and the restriction of $\Psi$ to $K$ is $\Phi_{1}+\cdots+\Phi_{m}$. Let $q_{K}(\tau, \Psi)$ denote the product of $p(\sigma, \Psi)$ for all injections $\sigma$ of $L$ into $\boldsymbol{C}$ which coincide with $\tau$ on $K$. Then $q_{K}(\tau, \Psi) / q_{K}(\tau \rho, \Psi)$ differs from (1.1) by an algebraic factor.

The proof will be given in $\S 5$.

Let $I_{K}^{0}\left(\right.$ resp. $\left.I_{K}^{0}(\boldsymbol{Q})\right)$ denote the submodule of $I_{K}$ (resp. $\left.I_{K}(\boldsymbol{Q})\right)$ consisting of all $\sum_{\tau} c_{\tau} \tau$ such that $c_{\tau}+c_{\tau \rho}$ does not depend on $\tau$. Notice that if $\Psi$ is a $C M$-type of an extension of $K$, the restriction of $\Psi$ to $K$ belongs to $I_{K}^{0}$. Now we state the stronger form of the above theorem as

THEOREM 1.3. Let $\Phi_{1}, \cdots, \Phi_{m}$ be CM-types of $K$ and suppose $\sum_{i=1}^{m} r_{i} \Phi_{i}=0$ with $r_{i} \in \boldsymbol{Q}$. Then, for every injection $\tau$ of $K$ into $\boldsymbol{C}$, we have 


$$
\Pi_{i=1}^{m}\left[p\left(\tau, \Phi_{i}\right) / p\left(\tau \rho, \Phi_{i}\right)\right]^{r_{i}} \sim 1 .
$$

Moreover, let $\Psi$ be a CM-type of an extension $L$ of $K$. Suppose the restriction of $\Psi$ to $K$ can be written $\sum_{i=1}^{m} s_{i} \Phi_{i}$ with $s_{i} \in \boldsymbol{Q}$. Then, for every injection $\tau$ of $K$ into $C$, we have

$$
q_{k}(\tau, \Psi) / q_{K}(\tau \rho, \Psi) \sim \prod_{i=1}^{m}\left[p\left(\tau, \Phi_{i}\right) / p\left(\tau \rho, \Phi_{i}\right)\right]^{s_{i}},
$$

where $q_{K}(\tau, \Psi)$ is defined as in Theorem 1.2.

PROOF. The first assertion can be derived from Theorem 1.2 by expressing the equality $\sum_{i} r_{i} \Phi_{i}=0$ as $\sum_{i} a_{i} \Phi_{i}=\sum_{i} b_{i} \Phi_{i}$ with positive integers $a_{i}$ and $b_{i}$. To prove the second assertion, observe that the restriction of $\Psi$ to $K$ can be written $\sum_{k=1}^{n} \Phi_{k}^{\prime}$ with $C M$-types $\Phi_{k}^{\prime}$ of $K$. Take positive integers $f, c_{i}$ and $d_{i}$ so that $f_{s_{i}}=c_{i}-d_{i}$. Then $f \sum_{k} \Phi_{k}^{\prime}+\sum_{i} d_{i} \Phi_{i}=\sum_{i} c_{i} \Phi_{i}$, so that the desired result follows from Theorem 1.2.

Let us now insert here a few propositions which can easily be derived from our definition.

Proposition 1.4. Let $(K, \Phi)$ and $(L, \Psi)$ be CM-types such that $K \subset L$ and the restriction of $\Psi$ to $K$ is $[L: K]$ times $\Phi$. Then $p(\tau, \Phi) \sim p(\sigma, \Psi)$ if $\tau$ is the restriction of $\sigma$ to $K$.

Proof. Let $(A, \iota)$ and $\left(A^{\prime}, \iota^{\prime}\right)$ be of type $(K, \Phi)$ and $(L, \Psi)$, respectively. By [7, Proposition 14], $A^{\prime}$ is isogenous to the product of $[L: K]$ copies of $A$. Our assertion follows immediately from this fact.

PROPOSITION 1.5. Let $(K, \Phi)$ be a CM-type, and $\sigma$ an isomorphism of a field $K_{1}$ onto $K$. Then $p(\sigma \tau, \sigma \Phi) \sim p(\tau, \Phi)$, where we let $\sigma$ act on $I_{K}$ on the left in an obvious way.

This is obvious.

The nature of the periods of the anti-holomorphic 1-forms $\bar{\omega}_{\nu}$ can be seen from

PROPOSITION 1.6. $p(\tau, \Phi)^{\rho} \sim p(\tau, \Phi)$.

ProOF. As shown in [13, pp. 381-383], the number $p(\tau, \Phi)$ can be obtained as the value $h\left(w_{0}\right)$ of a quotient $h=f_{1} / f_{2}$ of two Hilbert modular forms $f_{1}$ and $f_{2}$, with Fourier coefficients in $K_{0}^{\tau}$, at a point $w_{0}=\left(w^{\tau_{1}}, \cdots, w^{\tau} g\right)$ with $w \in K$ such that $\operatorname{Im}\left(w^{\tau_{\nu}}\right)>0$ for all $\tau_{\nu}$. We can take $w$ to be pure imaginary. Then $h\left(w_{0}\right)$ is real.

We have so far been interested in $p(\tau, \Phi)$ only up to algebraic factors. Let us now refine this point by considering them and their products up to factors belonging to smaller fields. For simplicity, let us assume that all number fields in the following discussion are subfields of $C$, and denote by $K_{a b}$ the maximal abelian extension of a number field $K$. For each $\Phi \in I_{K}$, let $K^{\Phi}$ denote the field generated over $\boldsymbol{Q}$ by $\operatorname{tr} \Phi(a)$ for all $a \in K$. Let $M$ be the Galois closure of $K$ over $\boldsymbol{Q}$, and let $G=\mathrm{Gal}(M / \boldsymbol{Q})$ and $H=\mathrm{Gal}(M / K)$. Then $I_{K}$ 
can be identified with the $Z$-module generated by the cosets $H \alpha$ with $\alpha \in G$; $G$ acts on $I_{K}$ on the right. It is easy to see that

$$
\left.\operatorname{Gal}\left(M / K^{\Phi}\right)=\{\gamma \in G \mid \Phi r=\Phi\} \quad \text { (cf. }[15, \S 8.3]\right) .
$$

Conjecture 1.7. Let $\tau$ be an injection of a $C M$-field $K$ into $\boldsymbol{C}$, and $\Phi \in I_{K}^{0}$. Then we can specify a non-zero complex number $P(\tau, \Phi)$, up to factors belonging to $\left(K^{\Phi}\right)_{a b} K_{0}^{\tau}$, with the property that if $\Phi=\sum_{i=1}^{m} s_{i} \Phi_{i}$ with CM-types $\Phi_{i}$ of $K$ and $s_{i} \in \boldsymbol{Z}$, then

$$
P(\tau, \Phi) \sim \prod_{i=1}^{m}\left[p\left(\tau, \Phi_{i}\right) / p\left(\tau \rho, \Phi_{i}\right)\right]^{s_{i}} .
$$

We now show that this conjecture can be reduced to a certain special case. Let $[K: \boldsymbol{Q}]=2 \mathrm{~g}$ and let $\Omega$ denote the sum of all injections of $K$ into C. Every element $\Phi$ of $I_{K}^{0}$ can be expressed as $\Phi=a \Omega+\boldsymbol{E}$ with $a \in \boldsymbol{Z}$ and

$$
\boldsymbol{E}=\sum_{\nu=1}^{g}\left[a_{\nu} \tau_{\nu}+\left(n-a_{\nu}\right) \tau_{\nu} \rho\right],
$$

where the $\tau_{\nu}$ are $g$ injections which form a $C M$-type of $K$, and the $a_{\nu}$ are non-negative integers such that $n-a_{g} \leqq a_{g} \leqq \cdots \leqq a_{1}=n$. Such $n$ is uniquely determined by $\Phi$. In view of Proposition 1.5, we can put $P(\tau, \Phi)=P(\tau, \boldsymbol{\Xi})$. Let us prove :

(1.2) Conjecture 1.7 is true if either $n$ is odd or $n=0$;

(1.3) Conjecture 1.7 is true if it is true for $n=2$.

First if $n=0$, we can put $P(\tau, \boldsymbol{\Xi})=1$. If $n=1, \Xi$ is a $C M$-type, and $P(\tau, \boldsymbol{\Xi})$ must be $\sim p\left(\tau_{\nu}, \boldsymbol{\Xi}\right)$ or $p\left(\tau_{\nu}, \boldsymbol{\Xi}\right)^{-1}$ according as $\tau=\tau_{\nu}$ or $\tau=\tau_{\nu} \rho$. Now $p\left(\tau_{\nu}, \boldsymbol{E}\right)$ can be given as the value $h(w)$ as explained in the proof of Proposition 1.6, which settles the problem, since the quotient of two such values belong to $\left(K^{\Xi}\right)_{a b} K_{0}^{\tau}$. If $n=2, \Xi$ has the form

$$
\Xi=\sum_{\nu=1}^{r} 2 \tau_{\nu}+\sum_{\nu=r+1}^{g}\left(\tau_{\nu}+\tau_{\nu} \rho\right)
$$

with $0<r \leqq g$. Let us assume that one can specify $P(\tau, \boldsymbol{E})$ for such $\boldsymbol{\Xi}$ as in our conjecture. Suppose $n>2$. If $a_{g}>n / 2$ (which is the case when $n$ is odd), $\Xi$ can be written

$$
\Xi=\sum_{j=1}^{t}\left[b_{j} \xi_{j}+\left(n-b_{j}\right) \xi_{j} \rho\right]
$$

with partial sums $\xi_{1}, \cdots, \xi_{t}$ of $\sum_{j=1}^{g} \tau_{\nu}$ such that $\sum_{j=1}^{t} \xi_{j}=\sum_{\nu=1}^{g} \tau_{\nu}$ and positive integers $b_{j}$ such that $n-b_{t}<b_{t}<\cdots<b_{1}=n$. Then we can find $C M$-types $\Phi_{1}, \cdots, \Phi_{n}$ such that $\Xi=\sum_{i=1}^{n} \Phi_{i}$ and each $\Phi_{i}$ has an expression $\Phi_{i}=\xi_{1}+\cdots+$ $\xi_{s}+\xi_{s+1} \rho+\cdots+\xi_{t} \rho$. Observe that if $\Xi \gamma=\Xi$ for an element $\gamma \in G$, then $\xi_{j} \gamma=\xi_{j}$ for every $j$, and hence $\Phi_{i} \gamma=\Phi_{i}$ for every $i$. This implies that $K^{\Phi_{i}} \subset K^{\Xi}$. Therefore we can put $P(\tau, \Xi)=\prod_{i=1}^{n} P\left(\tau, \Phi_{i}\right)$. Next suppose $a_{g}=n / 2$; then $n \geqq 4$. We are going to define $P(\tau, \Xi)$ by induction on $n$. We observe that

$$
\Xi=\sum_{\nu=1}^{r}\left[a_{\nu} \tau_{\nu}+\left(n-a_{\nu}\right) \tau_{\nu} \rho\right]+(n / 2) \sum_{\nu=r+1}^{g}\left(\tau_{\nu}+\tau_{\nu} \rho\right)
$$


with the integer $r$ such that $a_{r-1}>a_{r}=\cdots=a_{g}$. Put

$$
\Psi=\sum_{\nu=1}^{r} 2 \tau_{\nu}+\sum_{\nu=r+1}^{g}\left(\tau_{\nu}+\tau_{\nu} \rho\right)
$$

and $\Xi=\Psi+\Xi^{\prime}$. Then $\Xi \gamma=\Xi$ implies $\Psi \gamma=\Psi$ and $\Xi^{\prime} \gamma=\Xi^{\prime}$, and hence $K^{\Psi} K^{\Xi^{\prime}}$ $\subset K^{\Xi}$. By induction, $P(\tau, \Psi)$ and $P\left(\tau, \Xi^{\prime}\right)$ are meaningful. Therefore we can put $P(\tau, \Xi)=P(\tau, \Psi) P\left(\tau, \Xi^{\prime}\right)$.

We shall prove in $\S 7$ that if $\Xi$ is given by (1.4), $P\left(\tau_{\nu}, \Xi\right)$ can be specified as in our conjecture at least for $\nu \leqq r$. Thus the conjecture is reduced to the problem of finding $P\left(\tau_{\nu}, \Xi\right)$ for such $\Xi$ and for $\nu>r$.

\section{Applications and examples.}

The above theorems imply various interesting relations between the periods $p(\tau, \Phi)$. For simplicity, we assume in this section that all fields are subfields of $\boldsymbol{C}$. Let us begin with the simplest case where $K$ is an imaginary quadratic field. There are two $C M$-types $(K, \varphi)$ and $(K, \rho)$ with the identity map of $K$ as $\varphi$. The period in these cases is given by means of an elliptic modular form $h$ of weight 1 with algebraic Fourier coefficients. In fact, if $w$ is an element of $K$ such that $\operatorname{Im}(w)>0$ and $h(w) \neq 0$, we have $p(\varphi, \varphi) \sim$ $p(\rho, \rho) \sim h(w)$ by $[13$, Remark 3.4]. As a special case of Theorem 1.2, we obtain

Proposition 2.1. Let $(L, \Psi)$ be a CM-type. Suppose that $L$ contains an imaginary quadratic field $K$ and the restriction of $\Psi$ to $K$ is $r \varphi+s \rho$ with nonnegative integers $r$ and $s$, where $\varphi$ denotes the identity map of $K$. Then $q_{K}(\varphi, \Psi) / q_{K}(\rho, \Psi) \sim h(w)^{r-s}$.

A similar but somewhat different result has been obtained by Gross [2] with no mention of $p(\tau, \Phi)$ in the higher-dimensional case.

EXAMPLE 2.2. Suppose in particular $r=s$ in Proposition 2.1. Then we have $q_{K}(\varphi, \Psi) \sim q_{K}(\rho, \Psi)$ which is a non-trivial relation between the periods; this happens even when $(L, \Psi)$ is primitive. For instance, let $L_{0}$ be a totally real extension of $\boldsymbol{Q}$ of degree 4 with no subfields of degree 2, and let $L=L_{0} K$ with an imaginary quadratic field $K$. We can define a $C M$-type $\Psi=\sum_{\nu=1}^{4} \tau_{\nu}$ of $L$ so that $\tau_{1}=\tau_{2}=\varphi$ and $\tau_{3}=\tau_{4}=\rho$ on $K$. Then $(L, \Psi)$ is primitive and

$$
p\left(\tau_{1}, \Psi\right) p\left(\tau_{2}, \Psi\right) \sim p\left(\tau_{3}, \Psi\right) p\left(\tau_{4}, \Psi\right) .
$$

Similar examples can be obtained for fields of higher degree.

We now study the periods in connection with the reflex of a given $C M$ type. First, for a fixed $C M$-field $K$ of degree $2 g$, take a pair of $C M$-types $\Phi_{0}$ and $\Phi_{1}$ of the forms

$$
\Phi_{0}=\tau_{1}+\tau_{2}+\cdots+\tau_{g}, \quad \Phi_{1}=\tau_{1}+\left(\tau_{2}+\cdots+\tau_{g}\right) \rho,
$$


where $\tau_{1}$ is the identity map of $K$, and for each injection $\tau$ of $K$ into $\boldsymbol{C}$, define an element $\boldsymbol{p}_{K}(\tau)$ of $\boldsymbol{C}^{\times} / \overline{\boldsymbol{Q}}^{\times}$by

$$
\boldsymbol{p}_{K}(\tau) \sim\left\{\begin{array}{lll}
p\left(\tau_{1}, \Phi_{0}\right) p\left(\tau_{1}, \Phi_{1}\right) & \text { if } & \tau=\tau_{1}, \\
1 /\left[p\left[\tau_{1}, \Phi_{0}\right) p\left(\tau_{1}, \Phi_{1}\right)\right] & \text { if } & \tau=\rho, \\
p\left(\tau_{i}, \Phi_{0}\right) / p\left(\tau_{i} \rho, \Phi_{1}\right) & \text { if } & \tau=\tau_{i}, \\
p\left(\tau_{i} \rho, \Phi_{1}\right) / p\left(\tau_{i}, \Phi_{0}\right) & \text { if } & \tau=\tau_{i} \rho .
\end{array}\right.
$$

By Theorem 1.2, this definition does not depend on the choice of $\tau_{2}, \cdots, \tau_{g}$. Let $\Phi$ be an arbitrary $C M$-type of $K$, and $(J, \Psi)$ the reflex of $(K, \Phi)$ in the sense of $[11,1.3]$ (i. e., the dual defined in $[9,5.1]$ and $[15,8.3])$. Then $J=K^{\Phi}$. Let $M$ be the Galois closure of $K$ over $\boldsymbol{Q}$ and let $G=\operatorname{Gal}(M / \boldsymbol{Q})$. Consider $\Phi$ and $\Psi$ as the sets of injections of $K$ and $J$ into $M$. For each $\sigma \in \Psi$ take $\gamma \in G$ which gives $\sigma$ on $J$. Observe that $\Phi \gamma$ is a $C M$-type of $K$ depending only on $\sigma$, since $\Phi \alpha=\Phi$ for $\alpha \in \operatorname{Gal}(M / J)$; therefore we write $\Phi \gamma$ also $\Phi \sigma$.

THEOREM 2.3. If $(J, \Psi)$ is the reflex of $(K, \Phi)$ and $\sigma \in \Psi$, we have

$$
p(\sigma, \Psi)^{2} \sim \prod_{\tau \in \Phi \sigma} \boldsymbol{p}_{K}(\tau) .
$$

Proof. Observe that $\left(J^{\sigma}, \sigma^{-1} \Psi\right)$ is the reflex of $(K, \Phi \sigma)$. By Proposition 1.5 , we have $p(\sigma, \Psi) \sim p\left(1, \sigma^{-1} \Psi\right)$. Therefore, taking $\Phi \sigma$ and $\sigma^{-1} \Psi$ in place of $\Phi$ and $\Psi$, it is sufficient to prove

$$
p(1, \Psi)^{2} \sim \Pi_{\tau \in \Phi} \boldsymbol{p}_{K}(\tau)
$$

when $1 \in \Phi$ and $1 \in \Psi$. Since $M$ is a $C M$-field, we can define $\boldsymbol{p}_{M}(\alpha)$ for each $\alpha \in G$. We consider $\rho$ an element of $G$, which is actually contained in the center. For a subset $X$ of $G$, we use the same letter $X$ for the element of $I_{M}$ which is the sum of the elements of $X$. Let us now prove

$$
\prod_{\alpha \in X} \boldsymbol{p}_{M}(\alpha) \sim p\left(1,1+X^{-1}+Y\right) / p\left(1,1+X^{-1} \rho+Y\right)
$$

if $X$ and $Y$ are subsets of $G$ such that $1+X^{-1}+Y$ is a $C M$-type of $M$, where $X^{-1}=\left\{\gamma^{-1} \mid \gamma \in X\right\}$. This is obvious if $X$ is empty. Assume (2.4) is true for $X$ and let $W=X \cup\{\beta\}$; suppose $1+W^{-1}+Z$ is a $C M$-type. Then we have

$$
\begin{aligned}
\boldsymbol{p}_{M}(\beta) & \sim p\left(\beta, 1+\beta+\beta X^{-1}+\beta Z\right) / p\left(\beta \rho, 1+\beta \rho+\beta X^{-1} \rho+\beta Z \rho\right) \\
& \sim p\left(1,1+W^{-1}+Z\right) / p\left(1,1+X^{-1}+\beta^{-1} \rho+Z\right) .
\end{aligned}
$$

This multiplied by (2.4) with $Y=Z+\beta^{-1} \rho$ proves the case of $X \cup\{\beta\}$; thus the proof of (2.4) is completed by induction. Let $H=\operatorname{Gal}(M / K)$. Then $\boldsymbol{p}_{K}(H \beta)$ for $\beta \in G$ is meaningful. Now we have

$$
\boldsymbol{p}_{K}(H \beta) \sim \prod_{\alpha \in H \beta} \boldsymbol{p}_{M}(\alpha) .
$$


In fact, if $\beta \notin H$, we take a $C M$-type $\sum_{\nu=1}^{g} \tau_{\nu}$ of $K$ so that $\tau_{1}=\operatorname{id}_{K}$ and $\tau_{2}$ is represented by $H \beta$. Let $H \beta_{\nu}$ represent $\tau_{\nu}$ for $\nu>1$ with $\beta=\beta_{2}$. Then

$$
\begin{aligned}
\boldsymbol{p}_{K}\left(\tau_{2}\right) & \sim p\left(\tau_{2}, \tau_{1}+\tau_{2}+\cdots+\tau_{g}\right) / p\left(\tau_{2} \rho, \tau_{1}+\tau_{2} \rho+\cdots+\tau_{g} \rho\right) \\
& \sim p\left(\beta, H+H \beta_{2}+\cdots+H \beta_{g}\right) / p\left(\beta \rho, H+H \beta_{2} \rho+\cdots+H \beta_{g} \rho\right)
\end{aligned}
$$

by Proposition 1.4. Define $Y$ by $\beta Y+\beta=H \beta_{2}+\cdots+H \beta_{g}$. Then

$$
\begin{aligned}
\boldsymbol{p}_{K}\left(\tau_{2}\right) & \sim p(\beta, H+\beta+\beta Y) / p(\beta \rho, H+\beta \rho+\beta Y \rho) \\
& \sim p\left(1,1+\beta^{-1} H+Y\right) / p\left(1,1+\beta^{-1} H \rho+Y\right) \sim \prod_{\alpha \in H \beta} \boldsymbol{p}_{M}(\alpha)
\end{aligned}
$$

by (2.4); this proves $[2.5)$ in the case $\beta \notin H$. To prove the case $H \beta=H$, put $A=H-1$ and $B=H \beta_{2}+\cdots+H \beta_{g}$. Then

$$
\begin{aligned}
& \prod_{\alpha \in H} \boldsymbol{p}_{M}(\alpha) \sim \boldsymbol{p}_{M}(1) \prod_{\alpha \in A} \boldsymbol{p}_{M}(\alpha) \\
& \sim p(1,1+A+B \rho) p(1,1+A \rho+B) p(1,1+A+B) / p(1,1+A \rho+B) \quad(\text { by }(2.4)) \\
& \sim p(1, H+B) p(1, H+B \rho) \\
& \sim p\left(\tau_{1}, \tau_{1}+\cdots+\tau_{g}\right) p\left(\tau_{1}, \tau_{1}+\left(\tau_{2}+\cdots+\tau_{g}\right) \rho\right) \quad \text { (by Proposition 1.4) } \\
& \sim \boldsymbol{p}_{K}\left(\tau_{1}\right) .
\end{aligned}
$$

This completes the proof of (2.5), Now let $\Phi$ be represented by $H r_{1}, \cdots, H \gamma_{g}$, and let $S=H \gamma_{1}+\cdots+H \gamma_{g}=1+X$. By (2.4) and (2.5),

$$
\begin{aligned}
& \Pi_{\tau \in \Phi} \boldsymbol{p}_{K}(\tau) \sim \prod_{\alpha \in S} \boldsymbol{p}_{M}(\alpha) \sim \boldsymbol{p}_{M}(1) \prod_{\alpha \in X} \boldsymbol{p}_{M}(\alpha) \\
\sim & p\left(1,1+X^{-1}\right) p\left(1,1+X^{-1} \rho\right) p\left(1,1+X^{-1}\right) / p\left(1,1+X^{-1} \rho\right) \\
\sim & p\left(1,1+X^{-1}\right)^{2} \sim p\left(1, S^{-1}\right)^{2} .
\end{aligned}
$$

Now the restriction of $S^{-1}$ to $J$ is exactly $[M: J] \Psi$ according to the definition of the reflex. Thus $p\left(1, S^{-1}\right) \sim p(1, \Psi)$ by Proposition 1.4, and we obtain (2.2), This completes the proof.

REMARK 2.4. Let $\xi_{K}$ denote the set of all $C M$-types of $K$. Then $\xi_{K}$ has $2^{g}$ elements and $G$ acts on $\xi_{K}$ on the right. Let $\left\{\Phi_{1}, \cdots, \Phi_{s}\right\}$ be a complete set of representatives for $\mathfrak{F}_{K} / G$, and let $\left(J_{i}, \Psi_{i}\right)$ be the reflex of $\left(K, \Phi_{i}\right)$. Then $\left\{\Phi_{i} \gamma \mid \gamma \in G\right\}$ contains exactly $\left[J_{i}: \boldsymbol{Q}\right]$ elements, so that $\sum_{i=1}^{s}\left[J_{i}: \boldsymbol{Q}\right]=2^{g}$. Since the reflex of $\left(K, \Phi_{i} \gamma\right)$ is $\left(J_{i}{ }^{\gamma}, \gamma^{-1} \Psi_{i}\right)$, the $C M$-types $\left(J_{i}, \Psi_{i}\right)$, for $i=1, \cdots, s$, "represents" the reflexes of all the CM-types of $K$. Observe that $\boldsymbol{p}_{K}(\tau \rho)$ $=\boldsymbol{p}_{K}(\tau)^{-1}$. Therefore the above theorem shows that the $2^{g-1}$ numbers $p\left(\sigma, \Psi_{i}\right)^{2}$ are monomials of $g$ numbers $\boldsymbol{p}_{K}\left(\tau_{1}\right), \cdots, \boldsymbol{p}_{K}\left(\tau_{g}\right)$ and their inverses. As shown in $[11,1.9,1.10]$, it can happen that $s=1$ and $\left[J_{1}: Q\right]=2^{g}$. In any case, at most $g$ of the numbers $p\left(\sigma, \Psi_{i}\right)$ can be algebraically independent. We can refine this point as follows. 
THEOREM 2.5. Let $\Psi=\sum_{i=1}^{h} \sigma_{\lambda}$ be a CM-type of a CM-field $J$ of degree $2 h$, and $M$ the Galois closure of $J$ over $\boldsymbol{Q}$. Further let $t(\Psi)$ be the dimension of the svbspace of $I_{J}^{0}(\boldsymbol{Q})$ generated over $\boldsymbol{Q}$ by $\Psi \gamma$ for all $\gamma \in \operatorname{Gal}(M / \boldsymbol{Q})$, and let $\delta(\Psi)$ $=h+1-t(\Psi)$. Then the module

$$
\left\{\left(x_{1}, \cdots, x_{h}\right) \in Z^{h} \mid \Pi_{\lambda=1}^{h} p\left(\sigma_{\lambda}, \Psi\right)^{x} \lambda \sim 1\right\}
$$

has rank at least $\delta(\Psi)$.

Proof. We note that $\delta(\Psi) \geqq 0$, since $I_{J}^{0}(\boldsymbol{Q})$ has dimension $h+1$. Let $(K, \Phi)$ be the reflex of $(J, \Psi)$, and $\left(J^{\prime}, \Psi^{\prime}\right)$ the reflex of $(K, \Phi)$. Then the restriction of $\Psi$ to $J^{\prime}$ is $\left[J: J^{\prime}\right] \Psi^{\prime}$. Obviously $t(\Psi)=t\left(\Psi^{\prime}\right)$; therefore Proposition 1.4 reduces the problem to $\left(J^{\prime}, \Psi^{\prime}\right)$; so we assume $J=J^{\prime}$. Now by Kubota [17], we have

$$
t(\Psi)=t(\Phi)
$$

(This is non-trivial, but can be proved in an elementary way.) Changing $\Phi$ for $\Phi \rho$ if necessary, we may assume that $\rho \notin \Phi$. Let $W$ be the subspace of $I_{K}^{0}(\boldsymbol{Q})$ generated by $\Phi \sigma$ for all $\sigma \in \Psi$. Since $\Phi \gamma+\Phi \gamma \rho=\Phi+\Phi \rho$, we see that $\Phi \gamma \in W+\boldsymbol{Q} \Phi \rho$ for all $\gamma \in G$. Observe that $\Phi \rho \notin W$. Therefore $W$ has dimension $t(\Phi)-1$. If $\sum_{\lambda=1}^{h} x_{\lambda} \Phi \sigma_{\lambda}=0$, then Theorem 2.3 implies that

This proves our theorem.

$$
\Pi_{\lambda=1}^{h} p\left(\sigma_{\lambda}, \Psi\right)^{2 x \lambda} \sim 1 .
$$

REMARK 2.6. (a) Since $t(\Psi)=t\left(\Psi^{\prime}\right) \leqq \frac{1}{2}\left[J^{\prime}: \boldsymbol{Q}\right]+1$, we have $\delta(\Psi)=0$ only when $\Psi$ is primitive. The converse is not true, however.

(b) If $J$ is a Galois extension of $\boldsymbol{Q}$, it can easily be seen that $t(\Psi)$ is the dimension of the space generated over $\boldsymbol{Q}$ by $\gamma \Psi$ for all $\gamma \in \operatorname{Gal}(J / \boldsymbol{Q})$.

Proposition 2.7. Let $(K, \Phi)$ and $(L, \Psi)$ be CM-types. Suppose that $K \subset L$, $\delta(\Psi)=0$, and $L$ is a Galois extension of $\boldsymbol{Q}$. Then, for each injection $\tau$ of $K$ into $\boldsymbol{C}$, we have

$$
p(\tau, \Phi) \sim \prod_{\alpha \in G} p(\alpha, \Psi)^{b} \alpha
$$

with $b_{\alpha} \in \boldsymbol{Q}$, where $G=\operatorname{Gal}(L / \boldsymbol{Q})$.

PROof. There is a $C M$-type $\Psi_{1}$ of $L$ whose restriction to $K$ is $[L: K] \Phi$. Take $\beta \in G$ which gives $\tau$ on $K$. If $\delta(\Psi)=0, I_{L}^{0}(\boldsymbol{Q})$ is generated by $\alpha \Psi$ for all $\alpha \in G$, so that $\Psi_{1}=\sum_{\alpha \in G} c_{\alpha} \alpha \Psi$ with $c_{\alpha} \in \boldsymbol{Q}$. Then, for $\tau \in \Phi$,

$$
\begin{aligned}
p(\tau, \Phi) & \sim p\left(\beta, \Psi_{\mathrm{l}}\right) \sim \prod_{\alpha \in G}[p(\beta, \alpha \Psi) / p(\beta \rho, \alpha \Psi)]^{c_{\alpha}} \\
& \sim \prod_{\alpha \in G}\left[p\left(\alpha^{-1} \beta, \Psi\right) / p\left(\alpha^{-1} \beta \rho, \Psi\right)\right]^{c_{\alpha}}
\end{aligned}
$$

by Theorem 1.3 and Proposition 1.5. This proves our assertion.

REMARK 2.8. (a) It of ten happens that $I_{L}^{0}$ is generated over $Z$ by $\alpha \Psi$ for 
all $\alpha \in G$. In such a case, we have (2.7) with $b_{\alpha} \in Z$.

(b) The above proposition includes the case $K=L$. Therefore, when $L$ is normal over $\boldsymbol{Q}$ and $\delta(\Psi)=0$, the quantities $\boldsymbol{p}_{L}(\alpha)$ are algebraically dependent on $p(\alpha, \Psi)$ and vice versa.

Let us now assume that $K$ is an imaginary cyclic extension of $\boldsymbol{Q}$ of degree $2 g$. It can easily be seen that if $\Phi=\sum_{\nu=0}^{g-1} \sigma^{\nu}$ with a generator $\sigma$ of $\operatorname{Gal}(K / \boldsymbol{Q})$, we have $\delta(\Phi)=0$, and $\sigma^{\nu} \Phi$ for $\nu=0,1, \cdots, g$ form a basis of $I_{K}^{0}$ over $Z$. Let $(L, \Psi)$ be a $C M$-type such that $K \subset L$. Then the restriction of $\Psi$ to $K$ belongs to $I_{K}^{0}$, and hence. can be written $\sum_{\nu=0}^{g} s_{\nu} \sigma^{\nu} \Phi$ with $s_{\nu} \in Z$. By Theorem 1.3 and Proposition 1.5, we have

$$
\begin{array}{r}
q_{K}\left(\sigma^{\lambda}, \Psi\right) / q_{K}\left(\sigma^{\lambda+g}, \Psi\right) \sim \prod_{\nu=0}^{g}\left[p\left(\sigma^{\lambda-\nu}, \Phi\right) / p\left(\sigma^{\lambda+g-\nu}, \Phi\right)\right]_{\nu}^{s_{\nu}} \\
(\lambda=0,1, \cdots, g-1) .
\end{array}
$$

If the restriction of $\Psi$ to $K$ is $\sum_{\nu=0}^{2 g-1} r_{\nu} \sigma^{\nu}$ with $0 \leqq r_{\nu} \in Z$, we have $s_{0}=r_{0}, s_{1}=$ $r_{1}-r_{0}, \cdots, s_{g-1}=r_{g-1}-r_{g-2}$, and $s_{g}=r_{g}-r_{g-1}+r_{0}$. Consider (2.8) as "linear equations" in the variables $p\left(\sigma^{\mu}, \Phi\right)$ for $\mu=0,1, \cdots, g-1$. The determinant of the coefficients can be given in terms of $r_{\nu}$; if it is non-vanishing, we can express $p\left(\sigma^{\mu}, \Phi\right)$ by means of $q_{K}\left(\sigma^{\lambda}, \Psi\right)$. For example, if $g=2$, we obtain

Proposition 2.9. Let $K$ be an imaginary cyclic extension of $\boldsymbol{Q}$ of degree 4, and $\Phi=1+\sigma$ with a generator $\sigma$ of $\operatorname{Gal}(K / Q)$. Let $(L, \Psi)$ be a CM-type such that $K \subset L$, and let the restriction of $\Psi$ to $K$ be $\sum_{\nu=0}^{3} r_{\nu} \sigma^{\nu}$ with $0 \leqq r_{\nu} \in Z$. Suppose $r_{0} \neq r_{1}$ or $r_{1} \neq r_{2}$, and let

$$
a=\left(r_{1}-r_{2}\right) /\left[\left(r_{1}-r_{2}\right)^{2}+\left(r_{0}-r_{1}\right)^{2}\right], \quad b=\left(r_{0}-r_{1}\right) /\left[\left(r_{1}-r_{2}\right)^{2}+\left(r_{0}-r_{1}\right)^{2}\right] .
$$

Then we have

$$
\begin{aligned}
& p(1, \Phi) \sim q_{K}(1, \Psi)^{a} q_{K}\left(\sigma^{2}, \Psi\right)^{-a} q_{K}(\sigma, \Psi)^{-b} q_{K}\left(\sigma^{3}, \Psi\right)^{b}, \\
& p(\sigma, \Phi) \sim q_{K}(1, \Psi)^{b} q_{K}\left(\sigma^{2}, \Psi\right)^{-b} q_{K}(\sigma, \Psi)^{a} q_{K}\left(\sigma^{3}, \Psi\right)^{-a} .
\end{aligned}
$$

Observe that $r_{0}+r_{2}=r_{1}+r_{3}=[L: K]$. Therefore if $[L: K]$ is odd, we have $r_{0} \neq r_{1}$ or $r_{1} \neq r_{2}$.

Coming back to the case of an arbitrary degree, if equations (2.8) are not independent, we obtain non-trivial relations among $p(\tau, \Psi)$.

EXAMPLE 2.10. Suppose that $L$ is a cyclic extension of $\boldsymbol{Q}$ of degree 18, $[L: K]=3$, and $\tau$ a generator of $\operatorname{Gal}(L / Q)$ which coincides with $\sigma$ on $K$. Put $\Psi=1+\tau^{10}+\sum_{\nu=2}^{8} \tau^{\nu}$. Then $(L, \Psi)$ is primitive. In this case, the right-hand sides of (2.8) for $\lambda=0$ and $\lambda=2$ are the same; for $\lambda=1$, we obtain its inverse. Therefore

$$
\begin{aligned}
& p(1, \Psi) p\left(\tau^{6}, \Psi\right) / p\left(\tau^{3}, \Psi\right) \sim p\left(\tau^{4}, \Psi\right) p\left(\tau^{10}, \Psi\right) / p\left(\tau^{7}, \Psi\right) \\
& \quad \sim p\left(\tau^{2}, \Psi\right) p\left(\tau^{8}, \Psi\right) / p\left(\tau^{5}, \Psi\right) \sim p(1, \Phi) p\left(\sigma^{2}, \Phi\right) / p(\sigma, \Phi)
\end{aligned}
$$


In fact, we can easily verify that $\delta(\Psi)=2$, and these relations art those guaranteed by Theorem 2.5 and its proof.

The relations given by Proposition 2.7 can be used to express the periods in terms of the values of the gamma-function. For example, let $L=\boldsymbol{Q}(\zeta)$, $\zeta=\exp (2 \pi i / l)$ with an odd prime $l$. As shown by Weil [16], if $\Psi$ is the $C M$-type of $L$ obtained from a curve of the form $y^{l}=x^{a}(1-x), p(\alpha, \Psi)$ is the product of an algebraic number, a power of $\pi$, and a monomial of $\Gamma(t / l)$ for $t=1, \cdots, l-1$. It was shown by Kubota $[17]$ that $\delta(\Psi)=0$ for $a=1$. Therefore Proposition 2.7 guarantees an expression for $p(\tau, \Phi)$ in terms of $\Gamma(t / l)$ for every $C M$-type $\Phi$ of every imaginary subfield of $\boldsymbol{Q}(\zeta)$.

More $C M$-types of cyclotomic fields can be obtained from the factors of the jacobian of the curve $x^{d}+y^{e}=1$ with arbitrary positive integers $d$ and $e$. The periods of abelian integrals in the case $d=e$ have been computed by Rohrlich in [2, Appendix]. It seems that Theorem 1.3 and the idea in the proof of Proposition 2.7, together with a careful analysis of these $C M$-types, will lead to a general result about an expression of $p(\tau, \Phi)$ as a monomial of the values of $\Gamma$ for an arbitrary $C M$-type $\Phi$ of an arbitrary imaginary cyclotomic field, which will give a generalization of the formula of Chowla and Selberg in [6]. In the cases in which the periods are expressed by $\Gamma(t / l)$, our monomial relations can often be checked directly by means of the known relations of $\Gamma$ such as $\Gamma(s) \Gamma(1-s) \sim \pi$ for $s \in \boldsymbol{Q}$. Probably this is always so in such cyclotomic cases. In other words, it is unlikely that our relations produce new algebraic relations between the values of $\Gamma$.

Finally we mention that $p(\tau, \Phi)$ is closely connected with the values of $L$-functions of a $C M$-field with Hecke characters, as shown by [12, Theorem 2].

\section{The symmetric domain of a unitary group.}

To simplify our notation, we denote by $\operatorname{diag}\left[X_{1}, \cdots, X_{m}\right]$ the square matrix with square matrices $X_{1}, \cdots, X_{m}$ on the diagonal blocks and with 0 in all other blocks. If $S$ is a ring, we let $S_{q}^{p}$ denote the module of all $p \times q$ matrices with entries in $S$; we put $S^{p}=S_{1}^{p}$ and $M_{p}(S)=S_{p}^{p}$. For a complex hermitian matrix $H$, we write $H>0$ if $H$ is positive definite. Given two non-negative integers $r$ and $s$, put $J_{r, s}=\operatorname{diag}\left[1_{r},-1_{s}\right], m=r+s$, where $1_{r}$ denotes the identity matrix of degree $r$, and define a unitary group $U(r, s)$ by

$$
U(r, s)=\left\{\left.\alpha \in G L_{m}(\boldsymbol{C})\right|^{t} \bar{\alpha} J_{r, s} \alpha=J_{r, s}\right\}
$$

we understand that $J_{r, s}=1_{m}$ if $r s=0$. The quotient of $U(r, s)$ modulo a maximal compact subgroup is isomorphic to a bounded symmetric domain $\mathfrak{D}(r, s)$ defined by 


$$
\mathfrak{D}(r, s)=\left\{z \in \boldsymbol{C}_{s}^{r} \mid 1_{r}-z \cdot{ }^{t} \bar{z}>0\right\} .
$$

To define the action of $U(r, s)$ on $\mathscr{D}(r, s)$, let $\mathfrak{X}$ be the set of all elements $Y$ of $G L_{m}(\boldsymbol{C})$ such that ${ }^{t} \bar{Y} J_{r, s} Y=\operatorname{diag}[A,-B]$ with $0<A={ }^{t} \bar{A} \in \boldsymbol{C}_{r}^{r}$ and $0<B=$ ${ }^{t} \bar{B} \in C_{s .}^{s}$ Put

$$
Y(z)=\left[\begin{array}{ll}
1_{r} & z \\
t \bar{z} & 1_{s}
\end{array}\right] \quad(z \in \mathfrak{D}(r, s)) .
$$

It can easily be seen that

$$
(z, u, v) \mapsto Y(z)\left(\begin{array}{ll}
u & 0 \\
0 & v
\end{array}\right)
$$

gives a bijective map of $\mathfrak{D}(r, s) \times G L_{r}(\boldsymbol{C}) \times G L_{s}(\boldsymbol{C})$ onto $\mathfrak{X}$. Obviously $\alpha Y \in \mathfrak{X}$ if $\alpha \in U(r, s)$ and $Y \in \mathfrak{X}$. Therefore, if $z \in \mathfrak{D}(r, s)$, we can define a point $\alpha(z)$ of $\mathfrak{D}(r, s)$ by

$$
\alpha \cdot Y(z)=Y(\alpha(z)) \cdot \operatorname{diag}[\overline{\lambda(\alpha, z)}, \mu(\alpha, z)]
$$

with $\lambda(\alpha, z) \in G L_{r}(\boldsymbol{C})$ and $\mu(\alpha, z) \in G L_{s}(\boldsymbol{C})$. Notice that both $\lambda$ and $\mu$ are holomorphic factors of automorphy. Put

$$
R(z, w)={ }^{t} \overline{Y(z)} J_{r, s} Y(w)=\left[\begin{array}{cc}
1_{r}-z^{t} \bar{w} & w-z \\
{ }^{t} \bar{z}-{ }^{t} \bar{w} & { }^{t} \bar{z} w-1_{s}
\end{array}\right] .
$$

Substituting $\alpha(z)$ and $\alpha(w)$ for $z$ and $w$, we find

$$
\left.R(z, w)=\left[\begin{array}{cc}
t \lambda(\alpha, z) & 0 \\
0 & t
\end{array}\right] R(\alpha(z), z), \alpha(w)\right)\left[\begin{array}{cc}
\overline{\lambda(\alpha, w)} & 0 \\
0 & \mu(\alpha, w)
\end{array}\right] .
$$

Comparing the upper right blocks of both sides, we obtain

$$
d z \circ \alpha={ }^{t} \lambda(\alpha, z)^{-1} d z \mu(\alpha, z)^{-1} \quad(\alpha \in U(r, s)) .
$$

Put $\xi(z)=1_{r}-z \cdot{ }^{t} \bar{z}$ and $\eta(z)=1_{s}-{ }^{t} \bar{z} z$. Since $R(z, z)=\operatorname{diag}[\xi(z),-\eta(z)]$, (3.6) yields

$$
\xi(z){ }^{t} \lambda(\alpha, z) \xi(\alpha(z)) \overline{\lambda(\alpha, z)}, \quad \eta(z)={ }^{t} \overline{\mu(\alpha, z)} \eta(\alpha(z)) \mu(\alpha, z) .
$$

Now we can easily verify that $\operatorname{det}(Y(z))=\operatorname{det}(\xi(z))=\operatorname{det}(\eta(z))$. Taking the determinant of (3.4) and using (3.8), we find

$$
\operatorname{det}(\mu(\alpha, z))=\operatorname{det}(\alpha) \operatorname{det}(\lambda(\alpha, z)) \quad(\alpha \in U(r, s)) .
$$

If $r=0$ or $s=0$, the group $U(r, s)$ is compact. In this case, we denote by $\mathscr{D}(r, s)$ the space consisting of a single point, say $z_{0}$, and put $Y\left(z_{0}\right)=1_{m}$; the action of $U(r, s)$ on $\mathfrak{D}(r, s)$ is trivial. 


\section{A family of abelian varieties.}

Let $K$ be a $C M$-field, and $\Phi$ an element of $I_{K}$ which represents a class of representations of $K$. We consider a structure $(A, \iota, \mathcal{C})$ such that: (i) $(A, \iota)$ is of type $(K, \Phi)$; (ii) $\mathcal{C}$ is a polarization of $A$; and (iii) the involution of $\operatorname{End}(A) \otimes \boldsymbol{Q}$ determined by $\mathcal{C}$ sends $\iota(a)$ to $\iota\left(a^{o}\right)$. Take an isomorphism of $A$ onto a complex torus $C^{n} / D$ with a lattice $D$ in $C^{n}$ so that $\Phi(a)$ corresponds to $\iota(a)$, where we use $\Phi$ for a matrix representation in the given class. Then $K$ acts on $\boldsymbol{Q} D$ through $\Phi$, and hence there is an isomorphism $p$ of $K_{m}^{1}$ onto $\boldsymbol{Q} D$ such that $p(a x)=\Phi(a) p(x)$ for $a \in K$ and $x \in K_{m}^{1}$, where $m=2 n /[K: \boldsymbol{Q}]$. Put $\mathbb{M}_{\mathcal{C}}=p^{-1}(D)$. Then $\mathfrak{X}_{\mathcal{C}}$ is a lattice in $K_{m}^{1}$. Let $E(x, y)$ be the Riemann (alternating) form of a basic divisor in $\mathcal{C}$. As shown in [7], there is an element $T$ of $G L_{m}(K)$ such that ${ }^{t} T^{o}=-T$ and

$$
E(p(x), p(y))=\operatorname{Tr}_{K / \boldsymbol{Q}}\left(x T \cdot{ }^{t} y^{o}\right) \quad\left((x, y) \in K_{m}^{1} \times K_{m}^{1}\right) .
$$

In this situation, we say that $(A, \iota, \mathcal{C})$ is of type $\left(K, \Phi, T, \mathfrak{I}_{\mathcal{C}}\right)$, or simply of type $(K, \Phi, T)$ if only the isogeny-class is the question. Let $[K: \boldsymbol{Q}]=2 g$ and take $g$ injections $\tau_{1}, \cdots, \tau_{g}$ of $K$ into $C$ which form a $C M$-type of $K$; put $\Phi=\sum_{\nu=1}^{g}\left(r_{\nu} \tau_{\nu}+s_{\nu} \tau_{\nu} \rho\right)$ with integers $r_{\nu}$ and $s_{\nu}$. Then a structure of type $(K, \Phi, T)$ exists if and only if $-i T^{\tau_{\nu}}$ has signature $\left(r_{\nu}, s_{\nu}\right)$ for every $\nu$ (see [7]); especially $r_{1}+s_{1}=\cdots=r_{g}+s_{g}=m$, and hence $\Phi \in I_{K}^{0}$. Suppose in particular $\Phi$ is a $C M$-type and $\Phi=\sum_{\nu=1}^{g} \tau_{\nu}$. Then $m=1$ and $T$ is an element of $K$ such that $T^{\rho}=-T$ and $-i T^{\tau} \nu>0$ for all $\nu$. If $m>1$, the structures of a given type can be parametrized by the points of a symmetric domain $\mathfrak{D}$, as proved in [7]. Let us now recall this explicit parametrization.

With a fixed element $T$ of $G L_{m}(K)$ such that ${ }^{t} T^{\rho}=-T$, define a group $G_{Q}$ by

$$
G_{Q}=\left\{\alpha \in G L_{m}(K) \mid \alpha T \cdot{ }^{t} \alpha^{o}=T\right\} .
$$

Assuming the signature of $-i T^{\tau_{\nu}}$ to be $\left(r_{\nu}, s_{\nu}\right)$, take an element $Q_{\nu}$ of $G L_{m}(\overline{\boldsymbol{Q}})$ so that

$$
-i T^{\tau_{\nu}}=Q_{\nu}^{\rho} J_{r_{\nu}, s_{\nu}} \cdot{ }^{t} Q_{\nu} .
$$

Then $G_{\boldsymbol{Q}}$ can be embedded into $\Pi_{r=1}^{g} U\left(r_{\nu}, s_{\nu}\right)$ by the map

$$
\alpha \mapsto\left(Q_{1}^{-1} \alpha^{\tau_{1} \rho} Q_{1}, \cdots, Q_{g}{ }^{-1} \alpha^{\tau} g^{\rho} Q_{g}\right) .
$$

We let $G_{\boldsymbol{Q}}$ act on the domain

$$
\mathfrak{D}=\mathfrak{D}\left(r_{1}, s_{1}\right) \times \cdots \times \mathfrak{D}\left(r_{g}, s_{g}\right)
$$

through the map (4.3), Notice that $r_{\nu} s_{\nu}=0$ may happen for some $\nu$. For $\alpha \in G_{Q}$ and $z=\left(z_{1}, \cdots, z_{g}\right) \in \mathfrak{D}$ with $z_{\nu} \in \mathfrak{D}\left(r_{\nu}, s_{\nu}\right)$, we put $\lambda_{\nu}(\alpha, z)=\lambda\left(Q_{\nu}{ }^{-1} \alpha_{\nu}^{\tau}{ }_{\nu}^{\rho} Q_{\nu}, z_{\nu}\right)$, 
$\mu_{\nu}(\alpha, z)=\mu\left(Q_{\nu}{ }^{-1} \alpha^{\tau_{\nu} \rho} Q_{\nu}, z_{\nu}\right)$,

$$
Y_{\nu}(z)=Y\left(z_{\nu}\right)=\left\{\begin{array}{cc}
{\left[\begin{array}{ll}
1_{r_{\nu}} & z_{\nu} \\
t \bar{z}_{\nu} & 1_{s_{\nu}}
\end{array}\right]} & \left(r_{\nu} s_{\nu}>0\right), \\
1_{m} & \left(r_{\nu} s_{\nu}=0\right) .
\end{array}\right.
$$

Each point $z$ of $\mathfrak{D}$ determines a structure of type $(K, \Phi, T, \mathfrak{M})$ as follows. First put

$$
Y_{\nu}(z) \cdot{ }^{t} Q_{\nu}^{\rho}=\left[\begin{array}{lll}
u_{1}^{\nu} \cdots & u_{m}^{\nu} \\
\bar{v}_{1}^{\nu} & \cdots & \bar{v}_{m}^{\nu}
\end{array}\right] \quad(\nu=1, \cdots, g)
$$

with $u_{k}^{\nu} \in C^{r_{\nu}}$ and $v_{k}^{\nu} \in \boldsymbol{C}^{s_{\nu}}$, and define $\mathfrak{x}_{k}(z) \in \boldsymbol{C}^{m g}$ by

$$
{ }^{t} \mathfrak{x}_{k}(z)=\left({ }^{t} u_{k}^{1},{ }^{t} v_{k}^{1}, \cdots,{ }^{t} u_{k}^{g},{ }^{t} v_{k}^{g}\right) .
$$

Put $n=m g$, and define an $n$-dimensional diagonal representation $\Phi$ of $K$ by

$$
\Phi(a)=\operatorname{diag}\left[a^{\tau_{1}} 1_{r_{1}}, a^{\tau_{1} \rho} 1_{s_{1}}, \cdots, a^{\tau} g 1_{r_{g}}, a^{\tau g \rho} 1_{s_{g}}\right] \quad(a \in K) .
$$

For $a=\left(a_{1}, \cdots, a_{m}\right) \in K_{m}^{1}$ and $z \in \mathfrak{D}$, put

$$
p_{z}(a)=p(a, z)=\sum_{k=1}^{m} \Phi\left(a_{k}\right) \mathfrak{x}_{k}(z) .
$$

Then $p_{z}$ can be extended to an $\boldsymbol{R}$-linear isomorphism of $\left(K \otimes_{\boldsymbol{Q}} \boldsymbol{R}\right)_{m}^{1}$ onto $\boldsymbol{C}^{n}$. Therefore, if $\mathfrak{M}_{\mathfrak{C}}$ is a lattice in $K_{m}^{1}, \boldsymbol{C}^{n} / p_{z}(\mathfrak{M})$ is a complex torus. Define an $\boldsymbol{R}$-valued alternating form $E_{z}$ on $\boldsymbol{C}^{n}$ by

$$
E_{z}\left(p_{z}(a), p_{z}(b)\right)=\operatorname{Tr}_{K / \boldsymbol{Q}}\left(a T^{t} b^{o}\right) \quad\left(a, b \in K_{m}^{1}\right) .
$$

This defines a Riemann form on $\boldsymbol{C}^{n}$, so that the torus has a structure of abelian variety; the action of $\Phi(a)$ defines an endomorphism. In this way we obtain a structure $\left(A_{z}, \iota_{z}, \mathcal{C}_{z}\right)$ of type $\left(K, \Phi, T, \mathfrak{M}_{i}\right)$ for each $z \in \mathfrak{D}$. Conversely, every structure of type $(K, \Phi, T, \mathfrak{i})$ can be obtained in this fashion. For details, see [7].

We now define an embedding $\varepsilon$ of $\mathfrak{D}$ into the Siegel upper space

$$
\mathfrak{S}_{n}=\left\{\left.z \in \boldsymbol{C}_{n}^{n}\right|^{t} z=z, \operatorname{Im}(z)>0\right\} \text {. }
$$

First take a basis $\left\{h_{1}, \cdots, h_{2 n}\right\}$ of $K_{m}^{1}$ over $\boldsymbol{Q}$ so that

$$
\left(\operatorname{Tr}_{K / Q}\left(h_{i} T \cdot{ }^{t} h_{j}^{\rho}\right)\right)_{i, j=1, \ldots, 2 n}=\left[\begin{array}{cc}
0 & -1_{n} \\
1_{n} & 0
\end{array}\right],
$$

and define the period matrix $\left(\omega_{1}(z), \omega_{2}(z)\right)$ by

$$
\omega_{1}(z)=\left(p_{z}\left(h_{1}\right) \cdots p_{z}\left(h_{n}\right)\right), \quad \omega_{2}(z)=\left(p_{z}\left(h_{n+1}\right) \cdots p_{z}\left(h_{2 n}\right)\right) .
$$

As is well known, $\omega_{2}(z)$ is invertible, and $\omega_{2}(z)^{-1} \omega_{1}(z) \in \mathscr{H}_{n}$. Thus we obtain an embedding 


$$
\varepsilon: \mathfrak{D} \rightarrow \mathscr{H}_{n}, \quad \varepsilon(z)=\omega_{2}(z)^{-1} \omega_{1}(z) \quad(z \in \mathfrak{D}) .
$$

This is holomorphic, since $\mathfrak{x}_{k}(z)$ is holomorphic in $z$. Every $\beta=\left(\begin{array}{ll}A & B \\ C & D\end{array}\right) \in \operatorname{Sp}(n, \boldsymbol{R})$ acts on $\mathscr{S}_{n}$ as usual under the rule $\beta(Z)=(A Z+B)(C Z+D)^{-1}$ for $Z \in \mathfrak{S}_{n}$. Now, for every $\alpha \in G_{\boldsymbol{Q}}$, define an element $\alpha^{*}=\left(a_{i j}\right)$ of $G L_{2 n}(\boldsymbol{Q})$ by $h_{i} \alpha=\sum_{j=1}^{2 n} a_{i j} h_{j}$. Obviously $\alpha^{*} \in \operatorname{Sp}(n, \boldsymbol{Q})$. Moreover we have

$$
\varepsilon \circ \alpha=\alpha^{*} \circ \varepsilon \text {. }
$$

To show this, we first observe that, for $\alpha \in G_{Q}$,

$$
Y_{\nu}(z) \cdot{ }^{t} Q_{\nu}^{\rho} \cdot{ }^{t} \alpha^{\tau}{ }_{\nu}=\Lambda_{\nu}^{\prime}(\alpha, z) Y_{\nu}(\alpha(z)) \cdot{ }^{t} Q_{\nu}^{\rho},
$$

where

$$
A_{\nu}^{\prime}(\alpha, z)= \begin{cases}\operatorname{diag}\left[{ }^{t} \lambda_{\nu}(\alpha, z),{ }^{t} \mu_{\nu}(\alpha, z)^{o}\right] & \left(r_{\nu} s_{\nu}>0\right), \\ { }^{t}\left(Q_{\nu}^{-1} \alpha^{\tau}{ }_{\nu}{ }^{\rho} Q_{\nu}\right)^{\rho} & \left(r_{\nu} s_{\nu}=0\right) .\end{cases}
$$

Define $\Lambda(\alpha, z)$ and $\Lambda_{\nu}(\alpha, z)$ by

$$
\begin{gathered}
\Lambda(\alpha, z)=\operatorname{diag}\left[\Lambda_{1}(\alpha, z), \cdots, \Lambda_{g}(\alpha, z)\right], \\
\Lambda_{\nu}(\alpha, z)= \begin{cases}\operatorname{diag}\left[\lambda_{\nu}(\alpha, z), \mu_{\nu}(\alpha, z)\right] & \left(r_{\nu} s_{\nu}>0\right), \\
\left(Q_{\nu}^{-1} \alpha^{\tau}{ }_{\nu}^{\rho} Q_{\nu}\right)^{\rho} & \left(s_{\nu}=0\right), \\
Q_{\nu}^{-1} \alpha^{\tau}{ }^{\rho} Q_{\nu} & \left(r_{\nu}=0\right) .\end{cases}
\end{gathered}
$$

Then [4.9) implies that

$$
p(x \alpha, z)={ }^{t} \Lambda(\alpha, z) p(x, \alpha(z)) \quad\left(x \in K_{m}^{1}, z \in \mathfrak{D}\right) .
$$

Therefore we have

$$
\left(\omega_{1}(z), \omega_{2}(z)\right) \cdot{ }^{t} \alpha^{*}={ }^{t} \Lambda(\alpha, z)\left(\omega_{1}(\alpha(z)), \omega_{2}(\alpha(z))\right),
$$

which proves (4.8), At the same time we see that

$$
{ }^{t} \omega_{2}(\alpha(z)) \Lambda(\alpha, z) \cdot{ }^{t} \omega_{2}(z)^{-1}=C \cdot \varepsilon(z)+D \quad \text { if } \quad \alpha^{*}=\left(\begin{array}{ll}
A & B \\
C & D
\end{array}\right) .
$$

Let $\eta: G L_{n}(\boldsymbol{C}) \rightarrow G L_{p}(\boldsymbol{C})$ be a rational representation. Given a $\boldsymbol{C}_{q}^{p}$-valued function $f$ on $\mathscr{S}_{n}$, an element $\beta=\left(\begin{array}{ll}A & B \\ C & D\end{array}\right)$ of $\operatorname{Sp}(n, \boldsymbol{Q})$, and $k \in \boldsymbol{Z}$, define a $C_{q}^{p}$-valued function $\left.f\right|_{\eta, k} \beta$ by

$$
\left(\left.f\right|_{\eta, k} \beta\right)(Z)=\operatorname{det}(C Z+D)^{-k} \eta(C Z+D)^{-1} f(\beta(Z)) \quad\left(Z \in \mathscr{H}_{n}\right),
$$

and also a function $f^{\varepsilon}$ on $\mathscr{D}$ by

$$
f^{\varepsilon}(z)=\operatorname{det}\left(\omega_{2}(z)\right)^{-k} \eta\left({ }^{t} \omega_{2}(z)^{-1}\right) f(\varepsilon(z)) \quad(z \in \mathfrak{T}) .
$$

For every $\alpha \in G_{\boldsymbol{Q}}$, we have, by (4.11), 


$$
f^{\varepsilon}(\alpha(z))=\operatorname{det}(\Lambda(\alpha, z))^{k} \eta(\Lambda(\alpha, z))\left(\left.f\right|_{\eta, k} \alpha^{*}\right)^{s}(z) .
$$

Now define a congruence subgroup $\Gamma_{N}$ of $G_{Q}$ by

$$
\Gamma_{N}=\left\{\gamma \in G_{Q} \mid \operatorname{det}(\gamma)=1, \mathfrak{m} \gamma=\mathfrak{m}, \mathfrak{m}(1-\gamma) \subset N \mathfrak{m}\right\},
$$

where $N$ is a positive integer and $\mathfrak{m}=\sum_{j=1}^{2 n} \boldsymbol{Z} h_{j}$. The map $\alpha \mapsto \alpha^{*}$ sends $\Gamma_{N}$ into the group

$$
\Delta_{N}=\left\{\beta \in \operatorname{Sp}(n, \boldsymbol{Z}) \mid \beta \equiv 1_{2 n}(\bmod N)\right\} .
$$

Therefore, if $\left.f\right|_{\eta, k} \beta=f$ for all $\beta \in \Delta_{N}$, then $f^{\varepsilon}$ satisfies

$$
f^{\varepsilon}(\gamma(z))=\operatorname{det}(\Lambda(\gamma, z))^{k} \eta(\Lambda(\gamma, z)) f^{s}(z) \text { for all } \gamma \in \Gamma_{N} .
$$

The members of our family with complex multiplication can be obtained as follows (cf. Miyake [5]). Let

$$
Y=M_{n_{1}}\left(L_{1}\right) \oplus \cdots \oplus M_{n_{t}}\left(L_{t}\right)
$$

with $C M$-fields $L_{i}$ containing $K$, and let $\delta$ be a positive involution of $Y$. Suppose $m=\sum_{i=1}^{t} n_{i}\left[L_{i}: K\right]$ and there is a $K$-linear injection $h$ of $Y$ into $M_{m}(K)$ such that $h\left(x^{o}\right)=T \cdot{ }^{t} h(x)^{\rho} T^{-1}$ for all $x \in Y$. Put

$$
Y[\delta]=\left\{c \in Y \mid c c^{\delta}=1\right\} \text {. }
$$

Then $h(Y[\delta])$ is contained in $G_{Q}$ and has a unique common fixed point $z_{0}$ on D. There is a $\boldsymbol{Q}$-linear injection $\boldsymbol{\Xi}_{\nu}$ of $Y$ into $M_{m}(\boldsymbol{C})$, for each $\nu$, such that $\Xi_{\nu}(c)={ }^{t} \Lambda_{\nu}\left(h(c), z_{0}\right)$ for every $c \in Y[\delta]$. Moreover, there is a $C M$-type $\Psi_{i}$ of $L_{i}$ such that $\Psi_{i}=\sum_{\nu=1}^{g} \Psi_{i \nu}$ and $n_{i} \operatorname{tr}\left(\Psi_{i \nu}(a)\right)=\operatorname{tr}\left(\Xi_{\nu}(a)\right)$ for every $a \in L_{i}$. If $\left(A_{i}, \ell_{i}\right)$ is of type $\left(L_{i}, \Psi_{i}\right)$, the member $A_{z_{0}}$ is isogenous to $A_{1}{ }^{{ }} \times \cdots \times A_{t}{ }^{{ }}{ }$. Conversely, let $\left(L_{i}, \Psi_{i}\right)$ be $C M$-types such that $K \subset L_{i}$, and let $\left(A_{i}, \iota_{i}\right)$ be of type $\left(L_{i}, \Psi_{i}\right)$, Suppose the sum of the restrictions of $n_{1} \Psi_{1}, \cdots, n_{t} \Psi_{t}$ to $K$ is $\Phi$. Then $A=A_{1}{ }^{n_{1}} \times \cdots \times A_{t}{ }^{n_{t}}$ has a polarization which defines an involution of $\operatorname{End}(A) \otimes \boldsymbol{Q}$ whose restriction to the image of $K$ corresponds to $\rho$. As shown in [7], $A$ can be obtained as a member of a family of our type with suitable $T$ and $\mathfrak{M}_{i}$. We state here two facts about such special members as lemmas.

LEMMA 4.1. Let $\Phi_{1}, \cdots, \Phi_{m}$ be CM-types of $K$, and let $\left(A_{i}, \iota_{i}\right)$ be of type $\left(K, \Phi_{i}\right)$. If $\Phi=\sum_{i=1}^{m} \Phi_{i}$, the above family (without changing $T$ or $\mathfrak{i}$ ) contains a member isogenous to $A_{1} \times \cdots \times A_{m}$.

Proof. We can easily find (by induction on $m$, for example) elements $\zeta_{1}, \cdots, \zeta_{m}$ of $K$ such that $T$ is equivalent to $\operatorname{diag}\left[\zeta_{1}, \cdots, \zeta_{m}\right]$ and $\operatorname{Im}\left(\zeta_{i}^{\tau}\right)>0$ for $\tau$ belonging to $\Phi_{i}$, for every $i$. Then there is a structure $\left(A_{i}^{\prime}, \iota_{i}^{\prime}, \mathcal{C}_{i}^{\prime}\right)$ of type $\left(K, \Phi_{i}, \zeta_{i}\right)$ (see [15] or [7]), and $A_{1}^{\prime} \times \cdots \times A_{t}^{\prime}$ is isogenous to a member of our family.

Lemma 4.2. Suppose the member $A_{z}$ is isogenous to a product of abelian 
varieties belonging to CM-types. Then the coordinates of $\omega_{1}(z)$ and $\omega_{2}(z)$ are algebraic.

Proof. Observe that the action of $G_{\boldsymbol{Q}}$ on $\mathscr{D}$ is $\overline{\boldsymbol{Q}}$-rational. As explained above, $z$ is obtained as a unique fixed point of $Y[\delta]$. Therefore the coordinates of $z$ are algebraic, so that the vector $p_{z}(a)$ is algebraic for every $a \in K_{m}^{1}$, which proves our lemma.

\section{Proof of Theorem 1.2.}

The notation being as in Theorem 1.2, put $\Phi=\Phi_{1}+\cdots+\Phi_{m}$, and consider the family of $\S 4$ with this $\Phi$ and any choice of $T$ and $\mathfrak{X}$. If $\sum_{\nu=1}^{g} r_{\nu} s_{\nu}=0$, we have $\Phi_{1}=\cdots=\Phi_{m}$. In this case, Theorem 1.2 follows from Proposition 1.4. Therefore we may assume that $\mathfrak{D}$ is a domain of positive dimension.

Suppose first that either $\Gamma_{N} \backslash \mathfrak{D}$ is compact or $m g>2$. We denote then by $\mathfrak{A}_{0}\left(\Gamma_{N}\right)$ the field of all $\Gamma_{N}$-invariant meromorphic functions on $\mathscr{D}$, and by $\mathfrak{A}_{0}$ the union of $\mathfrak{A}_{0}\left(\Gamma_{N}\right)$ for all positive integers $N$. If $\Gamma_{N} \backslash \mathfrak{D}$ is not compact and $m g=2, \Gamma_{N}$ is conjugate to a congruence subgroup of $S L_{2}(\boldsymbol{Z})$; so we can similarly define $\mathfrak{A}_{0}\left(\Gamma_{N}\right)$ and $\mathfrak{A}_{0}$ under the usual cusp-condition. In either case, we can speak of canonical models, due to Miyake [5] (cf. also [8], [11]). Therefore $\overline{\boldsymbol{Q}}$-rational elements of $\mathfrak{A}_{0}$ are meaningful. We denote by $\mathfrak{A}_{0}(\overline{\boldsymbol{Q}})$ the subfield of $\mathfrak{A}_{0}$ consisting of all $\overline{\boldsymbol{Q}}$-rational elements. We note that if $f$ is a $\overline{\boldsymbol{Q}}$-rational Siegel modular function on $\mathfrak{H}_{n}$ and $f_{\circ} \varepsilon$ is meaningful, then $f \circ \varepsilon$ $\in \mathfrak{A}_{0}(\overline{\boldsymbol{Q}})$.

Let $P(Z)$ be a $\boldsymbol{C}_{n}^{n}$-valued meromorphic function on $\mathfrak{S}_{n}$ such that

$$
P(\beta(Z))=(C Z+D) P(Z) \quad \text { for every } \quad \beta=\left(\begin{array}{ll}
* & * \\
C & D
\end{array}\right) \in \Delta_{M}
$$

with a positive integer $M$. We can find such a $P$ which is " $Q$-rational", holomorphic at $\varepsilon(z)$, and with $\operatorname{det}(P(\varepsilon(z))) \neq 0$, for any given point $z$ of $\mathfrak{D}$, as proved in [13, Proposition 1.2]. Take a generator $b$ of $K$ over $\boldsymbol{Q}$ such that $b b^{o}=1$; put $\alpha=b 1_{m}, \alpha^{*}=\left(\begin{array}{cc}* & * \\ C & D\end{array}\right)$, and

$$
\begin{array}{ll}
V(Z)=P(Z)^{-1}(C Z+D)^{-1} P\left(\alpha^{*}(Z)\right) & \left(Z \in \mathfrak{S}_{n}\right), \\
U(z)=V(\varepsilon(z)) & (z \in \mathfrak{I}) .
\end{array}
$$

We see easily that the entries of $V$ are $\Delta_{N}$-invariant for some $N$. Moreover, they are $\bar{Q}$-rational by [13, Proposition 1.4]; hence the entries of $U$ belong to $\mathfrak{A}_{0}(\overline{\boldsymbol{Q}})$. By (4.12), we have

$$
{ }^{t} \omega_{2}(z) \Phi(b) \cdot{ }^{t} \omega_{2}(z)^{-1}=C \cdot \varepsilon(z)+D,
$$

and hence $P(\varepsilon(z))^{-1} \cdot{ }^{t} \omega_{2}(z) \Phi(b) \cdot{ }^{t} \omega_{2}(z)^{-1} P(\varepsilon(z))=U(z)^{-1}$. Since $K$ is generated by 
$b$, we see that

$$
a \mapsto P(\varepsilon(z))^{-1} \cdot{ }^{t} \omega_{2}(z) \Phi(a) \cdot{ }^{t} \omega_{2}(z)^{-1} P(\varepsilon(z))
$$

is an $\mathfrak{U}_{0}(\overline{\boldsymbol{Q}})$-rational representation of $K$, so that there is an element $Y$ of $G L_{n}\left(\mathfrak{A}_{0}(\overline{\mathbf{Q}})\right)$ such that

$$
P(\varepsilon(z))^{-1} \cdot{ }^{t} \omega_{2}(z) \Phi(a) \cdot{ }^{t} \omega_{2}(z)^{-1} P(\varepsilon(z))=Y^{-1} \Phi(a) Y
$$

for every $a \in K$. In view of our definition of $\Phi$ in the diagonal form, we have

$$
{ }^{t} \omega_{2}(z)^{-1} P(\varepsilon(z))=\operatorname{diag}\left[R_{1}, S_{1}, \cdots, R_{g}, S_{g}\right] Y
$$

with square matrices $R_{\nu}$ and $S_{\nu}$ of size $r_{\nu}$ and $s_{\nu}$, whose entries are meromorphic functions on $\mathfrak{D}$. From (4.16), we see that

$$
\begin{aligned}
& R_{\nu}(\gamma(z))= \begin{cases}\lambda_{\nu}(\gamma, z) R_{\nu}(z) & \left(r_{\nu} s_{\nu}>0\right), \\
\left(Q_{\nu}^{-1} \gamma^{\tau} \nu^{\rho} Q_{\nu}\right)^{\rho} R_{\nu}(z) & \left(s_{\nu}=0\right),\end{cases} \\
& S_{\nu}(\gamma(z))= \begin{cases}\mu_{\nu}(\gamma, z) S_{\nu}(z) & \left(r_{\nu} s_{\nu}>0\right), \\
Q_{\nu}^{-1} \gamma^{\tau}{ }^{\rho} Q_{\nu} S_{\nu}(z) & \left(r_{\nu}=0\right),\end{cases}
\end{aligned}
$$

if $\gamma \in \Gamma_{N}$, for sufficiently large $N^{\prime}$.

Let us now consider the special member at the fixed point $z_{0}$ of $h(Y[\delta])$ as in $\S 4$. For simplicity, we take $t=n_{1}=1$ and drop the subscript 1 ; thus the member at $z_{0}$ defines a structure $(A, \iota)$ of type $(L, \Psi)$ and $[L: K]=m$. (This is sufficient for our proof of Theorem 1.2. The following argument with obvious modifications applies to the general case.) Put $W=\varepsilon\left(z_{0}\right)$. Then $\boldsymbol{C}^{n} /\left(W \boldsymbol{Z}^{n}+\boldsymbol{Z}^{n}\right)$ is isogenous to $A$. Now take the projective embedding

$$
\Theta: \boldsymbol{C}^{n} /\left(W \boldsymbol{Z}^{n}+\boldsymbol{Z}^{n}\right) \rightarrow A_{0}
$$

of the torus onto an abelian variety $A_{0}$ by classical theta functions as defined in $\left[13,\left(1.5_{\mathrm{b}}\right)\right]$. Then $A_{0}$ is isogenous to $A$, and by $[13$, Theorem 1.1$]$, is defined over $\overline{\boldsymbol{Q}}$. Define an isomorphism

$$
\Theta^{\prime}: \boldsymbol{C}^{n} /\left[\omega_{1}\left(z_{0}\right) \boldsymbol{Z}^{n}+\omega_{2}\left(z_{0}\right) \boldsymbol{Z}^{n}\right] \rightarrow A_{0}
$$

by $\Theta^{\prime}(x)=\Theta\left(\omega_{2}\left(z_{0}\right)^{-1} x\right)$. Let $x_{1}, \cdots, x_{n}$ be the coordinate functions on $C^{n}$. By [13, Theorem 1.5], there are $\overline{\boldsymbol{Q}}$-rational 1 -forms $\xi_{1}, \cdots, \xi_{n}$ on $A_{0}$ such that

$$
\left[\begin{array}{c}
\xi_{1} \\
\vdots \\
\xi_{n}
\end{array}\right] \cdot \Theta^{\prime}=\pi \cdot t P\left(\varepsilon\left(z_{0}\right)\right) \omega_{2}\left(z_{0}\right)^{-1}\left[\begin{array}{c}
d x_{1} \\
\vdots \\
d x_{n}
\end{array}\right] .
$$

We have a direct sum decomposition of $C^{n}$ into subspaces $V_{\nu}, V_{\nu}^{\prime}$ such that $\Phi(a)$ acts as $a^{\tau} \nu$ on $V_{\nu}$ and as $a^{\tau} \rho^{\rho}$ on $V_{\nu}^{\prime}$. Let $x_{1}^{\nu}, \cdots, x_{r_{\nu}}^{\nu}$ and $y_{1}^{\nu}, \cdots, y_{s_{\nu}}^{\nu}$ be 
the coordinate functions on $V_{\nu}$ and $V_{\nu}^{\prime}$, respectively, which are renamings of some of $x_{1}, \cdots, x_{n}$. Multiplying (5.6) with a $\overline{\boldsymbol{Q}}$-rational matrix ${ }^{t} Y\left(z_{0}\right)^{-1}$, we find that

$$
\pi \cdot{ }^{t} R_{\nu}\left(z_{0}\right)\left[\begin{array}{c}
d x_{1}^{\nu} \\
\vdots \\
d x_{r}^{\nu}
\end{array}\right] \text { and } \pi \cdot{ }^{t} S_{\nu}\left(z_{0}\right)\left[\begin{array}{c}
d y_{1}^{\nu} \\
\vdots \\
d y_{s}^{\nu}
\end{array}\right] \quad\left(r=r_{\nu}, s=s_{\nu}\right)
$$

correspond to $\overline{\boldsymbol{Q}}$-rational 1 -forms on $A_{0}$.

Define diagonal representations $\Psi_{\nu}^{+}$and $\Psi_{\nu}^{-}$of $L$ by

$$
\Psi_{\nu}^{+}=\operatorname{diag}\left[\psi_{\nu 1}, \cdots, \phi_{\nu r}\right], \quad \Psi_{\nu}^{-}=\operatorname{diag}\left[\phi_{\nu 1}^{\prime}, \cdots, \phi_{\nu_{s}}^{\prime}\right]
$$

with injections $\phi_{\nu j}$ and $\phi_{\nu j}^{\prime}$ of $L$ into $C$ belonging to $\Psi$ which give $\tau_{\nu}$ and $\tau_{\nu} \rho$ on $K$, respectively. Then $r=r_{\nu}, s=s_{\nu}$, and $\Psi=\sum_{\nu_{=1}}^{g}\left(\Psi_{\nu}^{+}+\Psi_{\nu}^{-}\right)$. Take a generator $c$ of $L$ over $\boldsymbol{Q}$ such that $c c^{\rho}=1$. As explained in $\S 4, c \mapsto \Lambda_{\nu}\left(h(c), z_{0}\right)$ is essentially $\operatorname{diag}\left[\Psi_{\nu}^{+}(c), \Psi_{\nu}(c)\right]$; moreover, (if $r_{\nu} s_{\nu}>0$,) $\Psi_{\nu}^{+}(c)$ corresponds to $\lambda_{\nu}\left(h(c), z_{0}\right)$ and $\Psi_{\nu}^{-}(c)$ to $\mu_{\nu}\left(h(c), z_{0}\right)$. Therefore we can find invertible $\overline{\boldsymbol{Q}}$-rational matrices $C_{\nu}$ and $C_{\nu}^{\prime}$ such that

$$
\begin{aligned}
C_{\nu}^{-1} \Psi_{\nu}^{+}(c) C_{\nu} & = \begin{cases}\lambda_{\nu}\left(h(c), z_{0}\right) & \left(r_{\nu} s_{\nu}>0\right), \\
\left(Q_{\nu}^{-1} h(c)^{\tau}{ }_{\nu} \rho Q_{\nu}\right)^{\rho} & \left(s_{\nu}=0\right),\end{cases} \\
C_{\nu}^{\prime-1} \Psi_{\nu}^{-}(c) C_{\nu}^{\prime} & = \begin{cases}\mu_{\nu}\left(h(c), z_{0}\right) & \left(r_{\nu} s_{\nu}>0\right), \\
Q_{\nu}^{-1} h(c)^{\tau} \nu_{\nu} Q_{\nu} & \left(r_{\nu}=0\right) .\end{cases}
\end{aligned}
$$

Since ${ }^{t} \lambda_{\nu}\left(h(c), z_{0}\right)$ or $\left(Q_{\nu}^{-1} h(c)^{\tau} \nu^{\rho} Q_{\nu}\right)^{\rho}$ represents $\iota(c)$ on the subspace $V_{\nu}$, if we put

$$
\left[\begin{array}{c}
d u_{1}^{\nu} \\
\vdots \\
d u_{r}^{\nu}
\end{array}\right]={ }^{t} C_{\nu}^{-1}\left[\begin{array}{c}
d x_{1}^{\nu} \\
\vdots \\
d x_{r}^{\nu}
\end{array}\right] \quad\left(r=r_{\nu}\right)
$$

then $d u_{j}^{\nu}{ }^{\circ} \iota(c)=c^{\psi_{\nu j}} d u_{j}^{\nu}$. By Lemma 4,2, $d u_{j}^{\nu}$, considered a 1-form on $A_{0}$, has algebraic periods. By Proposition 1.1, $\pi \cdot p\left(\psi_{\nu j}, \Psi\right) d u_{j}$ is $\overline{\boldsymbol{Q}}$-rational, and hence

$$
\operatorname{diag}\left[p\left(\psi_{\nu 1}, \Psi\right), \cdots, p\left(\psi_{\nu r}, \Psi\right)\right]^{-1} C_{\nu} R_{\nu}\left(z_{0}\right) \in G L_{r}(\overline{\boldsymbol{Q}}),
$$

and similarly

$$
\operatorname{diag}\left[p\left(\psi_{\nu 1}^{\prime}, \Psi\right), \cdots, p\left(\psi_{\nu s}^{\prime}, \Psi\right)\right]^{-1} C_{\nu}^{\prime} S_{\nu}\left(z_{0}\right) \in G L_{s}(\overline{\boldsymbol{Q}}) .
$$

Put $f_{\nu}(z)=\operatorname{det}\left(R_{\nu}(z)\right)$ and $g_{\nu}(z)=\operatorname{det}\left(S_{\nu}(z)\right)$. Then we obtain

$$
f_{\nu}\left(z_{0}\right) \sim q_{K}\left(\tau_{\nu}, \Psi\right), \quad g_{\nu}\left(z_{0}\right) \sim q_{K}\left(\tau_{\nu} \rho, \Psi\right) .
$$

We can apply the whole procedure to a point of $\mathfrak{D}$ corresponding to a member of a somewhat different type. In fact, take $C M$-types $\Phi_{1}, \cdots, \Phi_{m}$ of $K$ so that $\Phi=\Phi_{1}+\cdots+\Phi_{m}$. Let $\left(A_{i}, \ell_{i}\right)$ be of type $\left(K, \Phi_{i}\right)$. Lemma 4.1 guar- 
antees a member of our family isogenous to $A_{1} \times \cdots \times A_{t}$. Let $v$ be a point of $D$ corresponding to this member. Since $v$ can be replaced by any point in the set $\left\{\alpha(v) \mid \alpha \in G_{Q}\right\}$ which is dense in $\mathscr{D}$, we may assume that the functions of (5.3) are all holomorphic at $v$ and $\operatorname{det}(P(\varepsilon(v))) \neq 0$. For each injection $\tau$ of $K$ into $C$, put $q(\tau)=\prod_{i=1}^{m} p\left(\tau, \Phi_{i}\right)$. Repeating the above reasoning with the direct sum of $m$ copies of $K$ in place of $L$, we find that $f_{\nu}(v) \sim q\left(\tau_{\nu}\right)$ and $g_{\nu}(v) \sim q\left(\tau_{\nu} \rho\right)$.

Suppose $r_{\nu} s_{\nu}>0$. By virtue of $\left(5.4_{\mathrm{a}, \mathrm{b}}\right)$ and (3.9), we see that $f_{\nu} / g_{\nu} \in \mathfrak{A}_{0}$, and $q\left(\tau_{\nu}\right)^{-1} q\left(\tau_{\nu} \rho\right)\left(f_{\nu} / g_{\nu}\right)(v) \in \overline{\boldsymbol{Q}}$. Now $v$ can be replaced by $\alpha(v)$ with $\alpha \in G_{\boldsymbol{Q}}$ if the functions of (5.3)] as well as $(P \circ \varepsilon)^{-1}$ are holomorphic at $\alpha(v)$. Since such points $\alpha(v)$ form a dense subset of $\mathscr{D}$, we see that $q\left(\tau_{\nu}\right)^{-1} q\left(\tau_{\nu} \rho\right) f_{\nu} / g_{\nu} \in \mathfrak{A}_{0}(\overline{\boldsymbol{Q}})$. Now $q\left(\tau_{\nu}\right) / q\left(\tau_{\nu} \rho\right)$ depends only on $\Phi_{1}, \cdots, \Phi_{m}$ and $\tau_{\nu}$. On the other hand, $f_{\nu} / g_{\nu}$ depends only on $\nu, \Phi, T$, and $\mathfrak{X}_{i}$. Therefore $q\left(\tau_{\nu}\right) / q\left(\tau_{\nu} \rho\right)$ depends only on $\Phi$ and $\tau_{\nu}$, which proves the first assertion of Theorem 1.2 in the case $r_{\nu} s_{\nu}>0$. Moreover, we have $q\left(\tau_{\nu}\right)^{-1} q\left(\tau_{\nu} \rho\right)\left(f_{\nu} / g_{\nu}\right)\left(z_{0}\right) \in \overline{\boldsymbol{Q}}$, and hence

$$
q\left(\tau_{\nu}\right) / q\left(\tau_{\nu} \rho\right) \sim f_{\nu}\left(z_{0}\right) / g_{\nu}\left(z_{0}\right) \sim q_{K}\left(\tau_{\nu}, \Psi\right) / q_{K}\left(\tau_{\nu} \rho, \Psi\right),
$$

which is the second assertion. If $s_{\nu}=0$, we see from $\left(5.4_{\mathrm{a}}\right)$ that $f_{\nu} \in \mathfrak{U}_{0}$, and conclude that $q\left(\tau_{\nu}\right)^{-1} f_{\nu} \in \mathfrak{A}_{0}(\overline{\boldsymbol{Q}})$ by the same reasoning as above. Therefore the above conclusions hold with $g_{\nu}=1$ and $q\left(\tau_{\nu} \rho\right)=q_{K}\left(\tau_{\nu} \rho, \Psi\right)=1$. The case $r_{\nu}=0$ can be treated in a similar way. This completes the proof.

\section{The derivatives of the elements of $\mathfrak{A}_{0}(\overline{\boldsymbol{Q}})$.}

The functions $R_{\nu}$ and $S_{\nu}$ obtained in $\S 5$ are (meromorphic) automorphic forms on $\mathfrak{D}$ with arithmetic properties $\left(5.9_{\mathrm{a}, \mathrm{b}}\right)$. Let us now study the relationship between $R_{\nu}, S_{\nu}$, and the derivatives of the elements of $\mathfrak{A}_{0}(\overline{\boldsymbol{Q}})$; namely, we prove:

THEOREM 6.1. Suppose $r_{\nu} s_{\nu}>0$ and let $z_{j k}^{\nu}$ be the $(j, k)$-entry of the variable $z_{\nu}$ on $\mathfrak{D}\left(r_{\nu}, s_{\nu}\right)$; define for $f \in \mathfrak{A}_{0}(\overline{\boldsymbol{Q}})$ a matrix-valued meromorphic function $\Delta_{\nu} f$ on $\mathbb{T}$ by

$$
\Delta_{\nu} f=\left(\partial f / \partial z_{j k}^{\nu}\right) \quad\left(1 \leqq j \leqq r_{\nu}, 1 \leqq k \leqq s_{\nu}\right) .
$$

Then there is a matrix $W$ with entries in $\mathfrak{A}_{0}(\overline{\boldsymbol{Q}})$ such that $\pi^{-1} \Delta_{\nu} f=R_{\nu} W \cdot{ }^{t} S_{\nu}$.

REMARK. (1) Our assertion with $\mathfrak{A}_{0}$ instead of $\mathfrak{A}_{0}(\overline{\boldsymbol{Q}})$ is obvious from (3.7) and $\left(5.4_{\mathrm{a}, \mathrm{b}}\right)$.

(2) Combining $\left(5.9_{\mathrm{a}, \mathrm{b}}\right)$ with the theorem, we obtain a description of the value of $\Delta_{\nu} f$ at a $C M$-point $z_{0}$ in terms of $p\left(\psi_{\nu j}, \Psi\right)$, similar to $(0.2)$,

ProOF. Let $\kappa=\sum_{\nu=1}^{g} r_{\nu} s_{\nu}$. We can find $\overline{\boldsymbol{Q}}$-rational Siegel modular functions $\mathfrak{f}_{1}, \cdots, \mathfrak{f}_{\kappa}$ on $\mathfrak{S}_{n}$ so that $\mathfrak{f}_{1}{ }^{\circ} \varepsilon, \cdots, \mathfrak{f}_{\kappa} \circ \varepsilon$ are algebraically independent. Put 
$k_{j}=\mathfrak{f}_{j}{ }^{\circ} \varepsilon$. Then $\partial / \partial h_{1}, \cdots, \partial / \partial h_{\kappa}$ are well-defined derivations of $\mathfrak{A}_{0}(\overline{\boldsymbol{Q}})$ and

$$
\Delta_{\nu} f=\sum_{j=1}^{\kappa}\left(\partial f / \partial h_{j}\right) \Delta_{\nu} h_{j} .
$$

Therefore it is suffcient to prove our theorem in the case where $f=f_{\circ} \circ$ with a $\overline{\boldsymbol{Q}}$-rational Siegel modular function $\mathfrak{f}$. To simplify the notation, we put $r_{1}=r, s_{1}=s$ and $\tau_{1}=\tau$, and prove our assertion for $\nu=1$. We may put $T$ in the form $T=\operatorname{diag}\left[\zeta_{1}, \cdots, \zeta_{m}\right]$ with $\zeta_{\lambda} \in K$ such that $i \zeta_{\lambda}^{\tau}<0$ for $\lambda \leqq r$ and $i \zeta_{\lambda}^{\tau}>0$ for $\lambda>r$. Take real numbers $q_{1}, \cdots, q_{m}$ so that $q_{\lambda}^{2}=-i \zeta_{\lambda}^{\tau}$ for $\lambda \leqq r$ and $q_{\lambda}^{2}=i \zeta_{\lambda}^{\tau}$ for $\lambda>r$. Then we can take $Q=\operatorname{diag}\left[q_{1}, \cdots, q_{m}\right]$ as the matrix $Q_{1}$ of (4.2). For each $\lambda$, choose bases $\left\{a_{\lambda 1}, \cdots, a_{\lambda g}\right\}$ and $\left\{a_{\lambda_{1}}^{\prime}, \cdots, a_{\lambda g}^{\prime}\right\}$ of $K_{0}$ over $\boldsymbol{Q}_{\mathbf{1}}$ so that

$$
\operatorname{Tr}_{K_{0} / \boldsymbol{Q}}\left(\zeta_{\lambda}^{2} a_{\lambda j} a_{\lambda k}^{\prime}\right)=\delta_{j k} / 2
$$

Let $\left\{e_{1}, \cdots, e_{m}\right\}$ be the standard basis of $K_{m}^{1}$, and let $h_{\lambda j}=a_{\lambda_{j}} e_{\lambda}$ and $h_{\lambda j}^{\prime}=$ $\zeta_{\lambda} a_{\lambda j}^{\prime} e_{\lambda}$. Then the elements

$$
h_{11}, \cdots, h_{1 g}, \cdots, h_{m 1}, \cdots, h_{m g}, h_{11}^{\prime}, \cdots, h_{1 g}^{\prime}, \cdots, h_{m 1}^{\prime}, \cdots, h_{m g}^{\prime}
$$

can be taken as $h_{1}, \cdots, h_{2 m g}$ of (4.5), Focusing our attention on the first $m$ rows of matrices, we have

$$
\omega_{1}(z)=\left(\begin{array}{cc}
b & z_{1} c \\
{ }^{t} z_{1} b & c \\
* & *
\end{array}\right), \quad \omega_{2}(z)=\left(\begin{array}{cc}
b^{\prime} & z_{1} c^{\prime} \\
-{ }^{t} z_{1} b^{\prime} & -c^{\prime} \\
* & *
\end{array}\right)
$$

with $b, b^{\prime} \in \boldsymbol{C}_{r g}^{r}$ and $c, c^{\prime} \in \boldsymbol{C}_{s g}^{s}$. Consider the functions on $D$ as functions in $z_{1}$, keeping the variables $z_{2}, \cdots, z_{g}$ constant; thus $d f=\sum_{j=1}^{r} \sum_{k=1}^{s}\left(\partial f / \partial z_{j k}^{1}\right) d z_{j k}^{1}$ $=\operatorname{tr}\left(\Delta_{1} f \cdot{ }^{t} d z_{1}\right)$. Since $d \varepsilon=\omega_{2}^{-1}\left(d \omega_{1}-d \omega_{2} \varepsilon\right)$, we have

$$
\begin{aligned}
& \omega_{2} d \varepsilon \cdot{ }^{t} \omega_{2}=d \omega_{1} \cdot{ }^{t} \omega_{2}-d \omega_{2} \cdot{ }^{t} \omega_{1} \\
& =\left(\begin{array}{ccc}
d z_{1}\left(c \cdot{ }^{t} c^{\prime}-c^{\prime} \cdot{ }^{t} c\right)^{t} z_{1} & -d z_{1}\left(c \cdot{ }^{t} c^{\prime}+c^{\prime} \cdot{ }^{t} c\right) & * \\
{ }^{t} d z_{1}\left(b \cdot{ }^{t} b^{\prime}+b^{\prime} \cdot{ }^{t} b\right) & { }^{t} d z_{1}\left(b^{\prime} \cdot{ }^{t} b-b \cdot{ }^{t} b^{\prime}\right) z & * \\
0 & 0 & 0
\end{array}\right) .
\end{aligned}
$$

This must be symmetric. The entries of $b, c, b^{\prime}, c^{\prime}$ can be given in terms of $q_{\lambda} a_{\lambda j}^{\tau}$ and $q_{\lambda} \zeta_{\lambda}^{\tau} a_{\lambda, j}^{\prime \tau}$. By virtue of (6.2), we find that

$$
\omega_{2} d \varepsilon \cdot{ }^{t} \omega_{2}=-i\left(\begin{array}{ccc}
0 & d z_{1} & 0 \\
{ }^{t} d z_{1} & 0 & 0 \\
0 & 0 & 0
\end{array}\right) .
$$


For a $\boldsymbol{Q}$-rational Siegel modular function $\mathfrak{f}$ on $\mathfrak{S}_{n}$, define a $\boldsymbol{C}_{n}^{n}$-valued function $\Delta \mathfrak{f}$ on $\mathscr{S}_{n}$ by

$$
\dot{\Delta}=\left(\frac{1+\delta_{j k}}{2} \frac{\partial \tilde{\mp}}{\partial Z_{j k}}\right) \quad\left(Z=\left(Z_{j k}\right) \in \mathscr{S}_{n}\right) .
$$

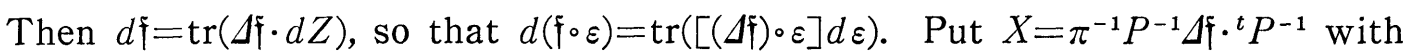
the function $P$ of (5.1). Then the entries of $X$ are $\overline{\boldsymbol{Q}}$-rational modular functions on $\mathscr{H}_{n}$. Consider $R_{\nu}, S_{\nu}$, and $Y$ of (5.3); put $V=Y \cdot(X \circ \varepsilon) \cdot{ }^{t} Y$ and $U=$ $\operatorname{diag}\left[R_{1}, S_{1}, \cdots, R_{g}, S_{g}\right]$. Then

$$
\pi^{-1} d(\mathfrak{f} \circ \varepsilon)=\operatorname{tr}\left(V \cdot{ }^{t} U \omega_{2} d \varepsilon \cdot{ }^{t} \omega_{2} U\right)=-2 i \cdot \operatorname{tr}\left(R_{1} v_{12} \cdot{ }^{t} S_{1} \cdot{ }^{t} d z_{1}\right),
$$

where $v_{12}$ is the $(1,2)$-block of $V$. Therefore $\pi^{-1} \Delta_{1}\left(\mathfrak{f}_{\circ} \varepsilon\right)=-2 i R_{1} v_{12} \cdot{ }^{t} S_{1}$, which completes the proof, since the entries of $v_{12}$ belong to $\mathfrak{A}_{0}(\overline{\boldsymbol{Q}})$.

\section{The derivatives of automorphic forms on $\mathfrak{Z}_{1}^{r}$.}

Let $F$ be a totally real algebraic number field of degree $g$, and $B$ a quaternion algebra over $F$. Let $\theta_{1}, \cdots, \theta_{g}$ denote the injections of $F$ into $\boldsymbol{R}$, and suppose that $B$ is unramified at $\theta_{1}, \cdots, \theta_{r}$, and ramified at $\theta_{r+1}, \cdots \theta_{g}$. Then there is an $\boldsymbol{R}$-linear isomorphism

$$
B \otimes_{\mathbf{Q}} \boldsymbol{R} \cong M_{2}(\boldsymbol{R})^{r} \times \boldsymbol{H}^{g-r}
$$

where $\boldsymbol{H}$ denotes the Hamilton quaternions. Let $B^{+}$denote the multiplicative group of all elements $\alpha$ of $B$ with totally positive reduced norm to $F$. We fix an isomorphism (7.1) so that $B$ is embedded in $M_{\mathbf{2}}(\overline{\boldsymbol{Q}} \cap \boldsymbol{R})^{r}$; then $B^{+}$can be embedded into $G L_{2}^{+}(\boldsymbol{R})^{r}$, where

$$
G L_{2}^{+}(\boldsymbol{R})=\left\{\alpha \in G L_{2}(\boldsymbol{R}) \mid \operatorname{det}(\alpha)>0\right\},
$$

and thus acts on the product $\mathfrak{S}_{1}^{r}$ of $r$ copies of the upper half plane $\mathfrak{H}_{1}$. With an arbitrarily fixed lattice $\mathfrak{a}$ in $B$ and a positive integer $N$, let

$$
E_{N}=\left\{\gamma \in B^{+} \mid \gamma \gamma^{\mathfrak{e}}=1, \gamma \mathfrak{a}=\mathfrak{a},(\gamma-1) \mathfrak{a} \subset N \mathfrak{a}\right\},
$$

where $\gamma \mapsto \gamma^{c}$ is the main involution of $B$. For $\alpha \in B$, let $\left(\alpha_{1}, \cdots, \alpha_{r}\right)$ denote the image of $\alpha$ in $M_{2}(\boldsymbol{R})^{r}$. Given $k=\left(k_{1}, \cdots, k_{r}\right) \in \boldsymbol{Z}^{r}, \alpha \in B^{+}$with $\alpha_{\nu}=\left(\begin{array}{ll}a_{\nu} & b_{\nu} \\ c_{\nu} & d_{\nu}\end{array}\right)$, and a function $f$ on $\mathscr{S}_{1}^{r}$, we define a function $\left.f\right|_{k} \alpha$ by

$$
\left(\left.f\right|_{k} \alpha\right)(z)=f(\alpha(z)) \Pi_{\nu=1}^{r}\left(c_{\nu} z_{\nu}+d_{\nu}\right)^{-k_{\nu}} .
$$

We denote by $\mathcal{A}_{k}\left(E_{N}\right)$ the set of all meromorphic functions $f$, meromorphic even at cusps, such that $\left.f\right|_{k} \gamma=f$ for all $\gamma \in E_{N}$; the cusp-condition is necessary only when $B=M_{2}(\boldsymbol{Q})$. Further we denote by $\mathcal{A}_{k}$ the union of $\mathcal{A}_{k}\left(E_{N}\right)$ for all $N$. Let $F^{\prime}$ denote the field generated over $\boldsymbol{Q}$ by $\sum_{\nu=1}^{r} a_{\nu}^{\theta_{\nu}}$ for all $a \in F$. 
The results of [10] and [11] enable us to speak of $\left(F_{a b}^{\prime}\right)$-rational elements of $A_{0}$. More generally, if $\Lambda$ is a subfield of $C$ containing $F_{a b}^{\prime}$, we can define the field $\mathcal{A}_{0}(\Lambda)$ consisting of $\Lambda$-rational elements of $A_{0}$.

Let $J$ be a totally imaginary quadratic extension of $F$ embeddable in $B$, and $h$ an $F$-linear injection of $J$ into $B$. Then $h\left(J^{\times}\right)$has a unique fixed point $w=\left(w_{1}, \cdots, w_{r}\right)$ on $\mathscr{S}_{1}^{r}$. Each $\theta_{\nu}$ can be extended to two injections of $J$ into C. If $\nu \leqq r$, we can specify one of them, say $\xi_{\nu}$, by

$$
h(a)_{\nu}\left[\begin{array}{c}
w_{\nu} \\
1
\end{array}\right]=\left[\begin{array}{c}
w_{\nu} \\
1
\end{array}\right] a^{\tilde{\xi}_{\nu}} \quad \text { for every } a \in J
$$

(Cf. $[9,2.6,2.7]$.) Thus $(J, h)$ determines $\left(J, \Xi_{0}\right)$ with $\Xi_{0}=\sum_{\nu=1}^{r} \xi_{\nu}$; this is a $C M$-type if $r=g$. Let $J^{\prime}$ denote the field generated over $\boldsymbol{Q}$ by $\operatorname{tr}\left(\boldsymbol{\Xi}_{0}(b)\right)$ for all $b \in J$. The main theorems of [10] and [11] assert

$$
f(w) \in J_{a b}^{\prime} \text { if } f \in \mathcal{A}_{0}\left(F_{a b}^{\prime}\right) \text { and } f \text { is finite at } w .
$$

Now the generalization of $(0.2)$ can be stated as

THEOREM 7.1. The notation $J, w$, and $\xi_{1}, \cdots, \xi_{r}$ being as above, take injections $\xi_{r+1}, \cdots, \xi_{g}$ of $J$ into $\boldsymbol{C}$ which extend $\theta_{r+1}, \cdots, \theta_{g}$, and put $\Xi_{1}=\sum_{\nu=1}^{g} \xi_{\nu}$ and $\Xi_{2}=$ $\sum_{\nu=1}^{r} \xi_{\nu}+\sum_{\nu=r+1}^{g} \xi_{\nu} \rho$. Let $f$ be an element of $\mathcal{A}_{0}(\overline{\boldsymbol{Q}})$ holomorphic at $w$. Then, for $1 \leqq \nu_{1}<\cdots<\nu_{s} \leqq r$, we have

$$
\left(\partial^{s} f / \partial z_{\nu_{1}} \cdots \partial z_{\nu_{s}}\right)(w) \sim \pi^{s} \prod_{i=1}^{s}\left[p\left(\xi_{\nu_{i}}, \Xi_{1}\right) p\left(\xi_{\nu_{i}}, \Xi_{2}\right)\right] .
$$

Notice that $p\left(\xi_{\nu}, \Xi_{1}\right) p\left(\xi_{\nu}, \Xi_{2}\right)$ for $\nu \leqq r$ depends only on $\xi_{1}, \cdots, \xi_{r}$, and is independent of the choice of $\xi_{r+1}, \cdots, \xi_{g}$, by virtue of Theorem 1.2. Before proving the theorem, we make some observations. First note that $\partial f / \partial z_{\nu} \neq 0$ for every $\nu \leqq r$ if $f$ is a non-constant element of $\mathcal{A}_{0}$.

PROPOSITION 7.2. Let $q_{\mu}=\left(\partial u / \partial z_{\mu}\right)^{-1} \partial v / \partial z_{\mu}$ with non-constant functions $u$ and $v$ of $\mathcal{A}_{0}\left(F_{a b}^{\prime}\right)$. Then $q_{\mu} \in \mathcal{A}_{0}(\overline{\boldsymbol{Q}})$. Moreover, $q_{\mu}(w) \in J_{a b}^{\prime} F_{\mu}^{\theta}$ for the fixed point $w$ of $h\left(J^{\times}\right)$as above, if $q_{\mu}$ is holomorphic at $w$.

Proof. Take a non-constant element $f$ of $\mathcal{A}_{0}\left(F_{a b}^{\prime}\right)$. Given $w$, we may assume, changing $f$ for $f \circ \beta$ with a suitable $\beta \in B^{+}$, that $\partial f / \partial z_{\mu}(w) \neq 0$ for every $\mu \leqq r$. Take $a \in J$ so that $J=\boldsymbol{Q}\left(a^{\rho} / a\right)$; put $\alpha=h(a), c_{\lambda}=\left(a^{\rho} / a\right)^{\lambda}$, and $s_{\lambda}=f \circ \alpha^{\lambda}$ for $\lambda=1, \cdots, r$. Then

$$
\frac{\partial\left(s_{1}, \cdots, s_{r}\right)}{\partial\left(z_{1}, \cdots, z_{r}\right)}(w)=\prod_{\mu=1}^{r} \frac{\partial f}{\partial z_{\mu}}(w) \cdot \operatorname{det}\left(c_{\lambda} \xi_{\nu}\right) \neq 0 .
$$

Therefore $s_{1}, \cdots, s_{r}$ are algebraically independent, and hence $\partial / \partial s_{1}, \cdots, \partial / \partial s_{r}$ are well-defined derivations of $\mathcal{A}_{0}\left(F_{a b}^{\prime}\right)$. Then $\partial u / \partial z_{\mu}=\Sigma_{\lambda}\left(\partial u / \partial s_{\lambda}\right)\left(\partial s_{\lambda} / \partial z_{\mu}\right)$, so that

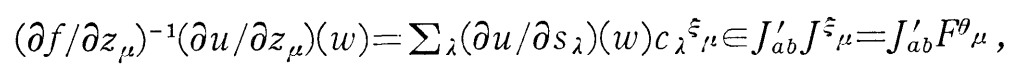


if $u$ is finite at $w$. Thus $q_{\mu}(w) \in J_{a b}^{\prime} F^{\theta \mu}$ whenever both $u$ and $v$ are finite at $w$ and $\left(\partial u / \partial z_{\mu}\right)(w) \neq 0$. Since such points $w$ with the same $J$ and $\left\{\hat{亏}_{1}, \cdots, \xi_{r}\right\}$ form a dense subset of $\mathfrak{S}_{1}^{r}$, we see the $q_{\mu} \in \mathcal{A}_{0}\left(J_{a b}^{\prime} F^{\vartheta^{\prime}}\right)$, which implies our assertions.

The above proof tempts us to make the following

CONJECTURE 7.3. (i) $\left(\partial u / \partial z_{\mu}\right)^{-1}\left(\partial v / \partial z_{\mu}\right) \in \mathcal{A}_{0}\left(F_{a b}^{\prime} F^{\theta^{\prime \prime}}\right)$ if $u, v \in \mathcal{A}_{0}\left(F_{a b}^{\prime}\right)$.

(ii) If $u$ is a non-constant function in $\mathcal{A}_{0}\left(E_{N}\right) \cap \mathcal{A}_{0}\left(F_{a b}^{\prime}\right)$, the divisor of $\partial u / \partial z_{\mu}$ on a canonical model of $E_{N} \backslash \mathfrak{S}_{1}^{r}$ is rational over $F_{a b}^{\prime} F^{\theta^{\prime}}$.

We now prove Theorem 7.1 in the case $s=1$, i.e.,

$$
\pi^{-1}\left(\partial f / \partial z_{\nu}\right)(w) \sim p\left(\xi_{\nu}, \Xi_{1}\right) p\left(\xi_{\nu}, \Xi_{2}\right) \quad \text { for } \quad \nu=1, \cdots, r .
$$

In view of Proposition 7.2, it is sufficient to prove this for one particular $f$ in $\mathcal{A}_{0}(\boldsymbol{Q})$. Take a totally imaginary quadratic extension $K$ of $F$, not isomorphic to $J$, which splits $B$. Identify $M_{2}(K)$ with $B \otimes_{F} K$. We can find an element $T$ of $G L_{2}(K)$ such that ${ }^{t} T^{o}=-T$ and

$$
B=\left\{\alpha \in M_{2}(K) \mid \alpha^{\imath} T=T \cdot{ }^{t} \alpha^{o}\right\},
$$

where $\iota$ denotes the main involution of $M_{2}(K)$. To show this, let $\gamma$ be the involution of $M_{2}(K)$ which coincides with $\rho$ on $K$ and with the main involution on $B$. Then $x^{\gamma}=u \cdot{ }^{t} x^{\rho} u^{-1}$ with a hermitian element $u$ of $G L_{2}(K)$. Putting $T=\zeta u$ with a pure imaginary element $\zeta$ of $K$, we obtain (7.7), We can easily verify that $i T^{\tau} \nu$ is indefinite or definite according as $\nu \leqq r$ or $\nu>r$ (cf. $[9,7.2]$, $[10,6.2])$. Define $G_{Q}$ of (4.1)] with this $T$. The group $\left\{\alpha \in B \mid \alpha \alpha^{\epsilon}=1\right\}$ is contained in $G_{\boldsymbol{Q}}$, and there is a holomorphic isomorphism $j$ of $\mathfrak{S}_{1}^{r}$ onto $\mathfrak{D}_{1}^{r}$ which makes the action of $\alpha$ on $\mathfrak{H}_{1}^{r}$ and on $\mathscr{D}_{1}^{r}$ compatible (cf. $[\mathbf{9},(7.3 .1)],[10,6.6]$ ). Moreover we have $\mathfrak{A}_{0}(\overline{\boldsymbol{Q}}) \circ j=\mathcal{A}_{0}(\overline{\boldsymbol{Q}})$, as our construction of canonical models in [10] and [11] shows; we note that $j$ is given by fractional linear transformations with algebraic coefficients.

Let $f \in \mathfrak{A}_{0}(\overline{\boldsymbol{Q}})$. If $w$ is the fixed point of $h\left(J^{\times}\right)$, we have, by Theorem 6.1,

$$
\left[\partial\left(\mathfrak{f}^{\circ} j\right) / \partial z_{1}\right](w) \sim \pi \cdot R_{1}(j(w)) S_{1}(j(w)),
$$

since the derivatives of $j$ at $w$ are algebraic. Note that $m=2$ and $r_{1}=s_{1}=1$ in the present case. Now take injections $\tau_{1}, \cdots, \tau_{g}$ of $K$ into $C$ so that $\tau_{\nu}=\theta_{\nu}$ on $F$ and $-i T^{\tau_{\nu}}>0$ for $\nu>r$. Then

$$
\Phi=\sum_{\nu=1}^{r}\left(\tau_{\nu}+\tau_{\nu} \rho\right)+\sum_{\nu=r+1}^{g} 2 \tau_{\nu}
$$

in the present case. The point $j(w)$ of $\mathfrak{D}$ corresponds to an abelian variety of type $(L, \Psi)$ with $L=J K$ and a $C M$-type $\Psi=\sum_{\nu=1}^{g}\left(\alpha_{\nu}+\beta_{\nu}\right)$ of $L$ defined by the table 


\begin{tabular}{|c|c|c|c|c|}
\hline & \multicolumn{2}{|c|}{$\nu \leqq r$} & \multicolumn{2}{|c|}{$\nu>r$} \\
\hline & on $J$ & on $K$ & on $J$ & on $K$ \\
\hline$\alpha_{\nu}=$ & $\xi_{\nu}$ & $\tau_{\nu}$ & $\xi_{\nu}$ & $\tau_{\nu}$ \\
\hline$\beta_{\nu}=$ & $\xi_{\nu}$ & $\tau_{\nu} \rho$ & $\xi_{\nu} \rho$ & $\tau_{\nu}$ \\
\hline
\end{tabular}

as shown in $[9,6.5]$ and $[\mathbf{1 0}, 6.8]$. By $[5.10)$ and Theorem 1.2, we have

$$
R_{1}(j(w)) S_{1}(j(w)) \sim p\left(\alpha_{1}, \Psi\right) p\left(\beta_{1}, \Psi\right) \sim p\left(\xi_{1}, \Xi_{1}\right) p\left(\xi_{1}, \Xi_{2}\right),
$$

which combined with (7.8) proves (7.6).

Let us now prove the statement at the end of $\S 1$. Put $\Xi=\Xi_{1}+\Xi_{2}$ and observe that $J^{\prime}=J^{z}$. By Proposition 7.2, the left-hand side of (7.6) is determined up to factors belonging to $J_{a b}^{\prime} F^{\theta_{\nu}}$. Therefore we can put $P\left(\tau_{\nu}, \boldsymbol{\Xi}\right)=$ $\pi^{-1}\left(\hat{\partial} f / \partial z_{\nu}\right)(w)$ with non-constant $f \in \mathcal{A}_{0}\left(F_{a b}^{\prime}\right)$ for each $\nu \leqq r$, which has the desired property of Conjecture 1.7. It should be noted that $\left(\partial f / \partial z_{\nu}\right)(w)$ depends on the choice of the algebra $B$ in which $J$ is embedded and also of the isomorphism (7.1),

The existence of " $\overline{\boldsymbol{Q}}$-rational forms of weight 1 " can be shown by

PROPOSITION 7.4. For each $\mu \leqq r$, there is a non-zero element $t$ of $\mathcal{A}_{k}$ such that $\pi t^{2}=u \cdot \partial f / \partial z_{\mu}$ with $u$ and $f$ in $\mathcal{A}_{0}(\overline{\boldsymbol{Q}})$, where $k$ is determined by $\mu$ in an obvious way.

Proof. The notation being as in the proof of (7.6), we have proved in $\S 5$ that $\delta R_{1} / S_{1} \in \mathfrak{U}_{0}(\overline{\boldsymbol{Q}})$ with $\delta=q\left(\tau_{1} \rho\right) / q\left(\tau_{1}\right)$. As already remarked, we have $j\left(z_{1}, \cdots, z_{r}\right)=\left(\left(a_{1} z_{1}+b_{1}\right) /\left(c_{1} z_{1}+d_{1}\right), \cdots\right)$ with $\left(\begin{array}{ll}a_{1} & b_{1} \\ c_{1} & d_{1}\end{array}\right)$ in $S L_{2}(\overline{\boldsymbol{Q}})$. Then $t=$ $\delta^{1 / 2}\left(R_{1} \circ j\right) /\left(c_{1} z_{1}+d_{1}\right)$ has the required property for $\mu=1$, by virtue of Theorem 6.1 .

Let $\mathcal{A}_{k}(\overline{\boldsymbol{Q}})$ denote, for $k=\left(k_{1}, \cdots, k_{r}\right) \in \boldsymbol{Z}^{r}$, the set of elements $\varphi$ of $\mathcal{A}_{\boldsymbol{k}}$ such that $\varphi^{2}=\pi^{-\{k\}} f \prod_{\nu=1}^{r}\left(\partial u / \partial z_{\nu}\right)^{k}$, with a fixed non-constant element $u$ of $\mathcal{A}_{0}\left(F_{a b}^{\prime}\right)$ and an arbitrary $f$ of $\mathcal{A}_{0}(\overline{\mathbf{Q}})$, where $\{k\}=\sum_{\nu=1}^{r} k_{\nu}$. Proposition 7.4 shows that $\mathcal{A}_{k}(\overline{\mathbf{Q}}) \neq\{0\}$ for every $k$; obviously $\mathcal{A}_{k}(\overline{\mathbf{Q}})$ is a one-dimensional vector space over $\mathcal{A}_{0}(\overline{\boldsymbol{Q}})$. By Proposition 7.2, $\mathcal{A}_{k}(\overline{\boldsymbol{Q}})$ does not depend on the choice of $u$. This implies in particular

Proposition 7.5. If $v \in \mathcal{A}_{k}(\overline{\boldsymbol{Q}})$ and $\alpha \in B^{+}$, then $\left.v\right|_{k} \alpha \in \mathcal{A}_{k}(\overline{\boldsymbol{Q}})$.

For $k=\left(k_{1}, \cdots, k_{r}\right)$ and $e=\left(e_{1}, \cdots, e_{r}\right)$ in $\boldsymbol{Z}^{r}$ with $e_{\nu} \geqq 0$, define a differential operator $\boldsymbol{d}_{k}^{e}$ of degree $\{e\}$ acting on the functions on $\mathscr{S}_{1}^{r}$ by

$$
\boldsymbol{d}_{k}^{e}=(2 \pi i)^{-\{\ell \mid} \prod_{\nu=1}^{r}\left(\frac{k_{\nu}+2 e_{\nu}-2}{z_{\nu}-\bar{z}_{\nu}}+\frac{\partial}{\partial z_{\nu}}\right) \cdots\left(\frac{k_{\nu}+2}{z_{\nu}-\bar{z}_{\nu}}+\frac{\partial}{\partial z_{\nu}}\right)\left(\frac{k_{\nu}}{z_{\nu}-\bar{z}_{\nu}}+\frac{\partial}{\partial z_{\nu}}\right),
$$

where $\{e\}=\sum_{\nu=1}^{r} e_{\nu}$; we understand that $\boldsymbol{d}_{k}^{0}$ is the identity operator (cf. $[12$, 
$\S 1])$.

THEOREM 7.6. Let $f \in \mathcal{A}_{k}(\overline{\mathbf{Q}})$ and $v \in \mathcal{A}_{k+2 e}(\overline{\boldsymbol{Q}})$ with $e_{\nu} \geqq 0$ for all $\nu$. Suppose that both $f$ and $v^{-1}$ are holomorphic at the fixed point $w$ of $h\left(J^{\times}\right)$as above. Then $\left(v^{-1} \boldsymbol{d}_{k}^{e} f\right)(w) \in \overline{\boldsymbol{Q}}$.

This is a generalization of the main theorem I of [12] and can be proved in exactly the same fashion.

COROLlaRY 7.7. If $f \in \mathcal{A}_{0}(\overline{\mathbf{Q}})$ and $0<\nu_{1}<\cdots<\nu_{s} \leqq r$, then

$$
\pi^{-s} \partial^{s} f / \partial z_{\nu_{1}} \cdots \partial z_{\nu_{s}} \in \mathcal{A}_{k}(\overline{\mathbf{Q}}),
$$

where $k$ is determined by $\nu_{1}, \cdots, \nu_{s}$ in an obvious way.

This follows immediately from Theorem 7.6, and proves, combined with (7.6), the general case of Theorem 7.1.

The above results concern the archimedean primes of $F$ unramified in $B$. Let us now prove an analogue of Proposition 7.4 for the remaining primes. Let $\chi_{\nu}$ for $\nu=r+1, \cdots, g$ be injections of $B$ into $M_{2}(\overline{\boldsymbol{Q}})$ such that $\chi_{\nu}(a)=a_{\nu}^{\theta_{\nu} 1_{2}}$ for $a \in F$. We consider $M_{2}(\boldsymbol{C})$-valued meromorphic functions $t$ on $\mathscr{2}_{1}^{r}$ such that

$$
\mathrm{t}(\gamma(z))=\chi_{\nu}(\gamma) \mathrm{t}(z) \quad \text { for every } \gamma \in E_{N}
$$

with some $N$. The existence of such $t$ can be shown as follows. Identifying $B$ with a subalgebra of $M_{2}(K)$, we can find an element $U_{\nu}$ of $G L_{2}(\overline{\boldsymbol{Q}})$ such that $U_{\nu}^{-1} \chi_{\nu}(a) U_{\nu}=\left(Q_{\nu}^{-1} a^{\tau}{ }_{\nu}^{\rho} Q_{\nu}\right)^{\rho}$ for all $a \in B$. Put $\mathrm{t}=U_{\nu} R_{\nu} \circ j$ with the function $R_{\nu}$ of (5.3) for a fixed $\nu>r$. Then (5.4 ) implies (7.10). Moreover, for any point $v$ of $\mathscr{S}_{1}^{r}$, we can take $\mathrm{t}$ so that $\mathrm{t}$ is holomorphic at $v$ and $\operatorname{det}(\mathrm{t}(v)) \neq 0$.

To define the $\overline{\boldsymbol{Q}}$-rationality of t, we consider the fixed point $w$ of $h\left(J^{\times}\right)$ as before, and define $\xi_{\nu}, \Xi_{1}$ and $\Xi_{2}$ as in Theorem 7.1. Then there is an element $D_{\nu}$ of $G L_{2}(\overline{\boldsymbol{Q}})$ such that

$$
D_{\nu} \chi_{\nu}(h(x)) D_{\nu}^{-1}=\operatorname{diag}\left[x^{\xi_{\nu} \rho}, x^{\xi_{\nu}}\right] \quad \text { for all } x \in J .
$$

Put $q_{\nu}=\left[p\left(\xi_{\nu}, \Xi_{1}\right) / p\left(\xi_{\nu} \rho, \Xi_{2}\right)\right]^{1 / 2}$. We now consider the following property of $t$.

$$
\operatorname{diag}\left[q_{\nu}, q_{\nu}^{-1}\right] D_{\nu} \mathrm{t}(w) \in M_{2}(\overline{\mathbf{Q}}) .
$$

Observe that this property does not depend on the choice of $D_{\nu}$.

PROPOSITION 7.8. For each $\nu>r$, there exists an $M_{2}(\boldsymbol{C})$-valued meromorphic function $\mathrm{t}$ on $\mathfrak{S}_{1}^{r}$ whose determinant is not identically equal to 0 , and which satisfies (7.10) for some $N$ and (7.12) at every fixed point $w$ of the above type where $\mathrm{t}$ is holomorphic.

Proof. The notation being as in the proof of (7.6), put $z_{0}=j(w)$ with the fixed point $w$ of $h\left(J^{\times}\right)$. Define $\Psi=\sum_{\nu=1}^{g}\left(\alpha_{\nu}+\beta_{\nu}\right)$ by (7.9). Since 


$$
D_{\nu} U_{\nu}\left(Q_{\nu}^{-1} h(x)^{\sigma_{\nu} \rho} Q_{\nu}\right)^{\rho} U_{\nu}^{-1} D_{\nu}^{-1}=\operatorname{diag}\left[x^{\xi_{\nu} \rho}, x^{\xi_{\nu}}\right] \quad \text { for } \quad x \in J,
$$

we can take $D_{\nu} U_{\nu}$ as $C_{\nu}$ of $\left(5.8_{\mathrm{a}}\right)$; hence we have, by $\left(5.9_{\mathrm{a}}\right)$,

$$
\operatorname{diag}\left[p\left(\beta_{\nu}, \Psi\right), p\left(\alpha_{\nu}, \Psi\right)\right]^{-1} D_{\nu} U_{\nu} R_{\nu}\left(z_{0}\right) \in G L_{2}(\overline{\mathbf{Q}}) .
$$

By Theorem 1.2, (if $\nu>r$,) we have

$$
\begin{aligned}
& p\left(\alpha_{\nu}, \Psi\right) / p\left(\beta_{\nu}, \Psi\right) \sim p\left(\xi_{\nu}, \Xi_{1}\right) / p\left(\xi_{\nu} \rho, \Xi_{2}\right), \\
& p\left(\alpha_{\nu}, \Psi\right) p\left(\beta_{\nu}, \Psi\right) \sim p\left(\tau_{\nu}, \Phi_{1}\right) p\left(\tau_{\nu}, \Phi_{2}\right),
\end{aligned}
$$

where $\Phi_{1}=\sum_{\nu=1}^{g} \tau_{\nu}$ and $\Phi_{2}=\sum_{\nu=1}^{r} \tau_{\nu} \rho+\sum_{\nu=r+1}^{g} \tau_{\nu}$. Therefore, if we put $b=$ $\left[p\left(\tau_{\nu}, \Phi_{1}\right) p\left(\tau_{\nu}, \Phi_{2}\right)\right]^{1 / 2}$ and $\mathrm{t}=b^{-1} U_{\nu} R_{\nu} \circ j$, then $\mathrm{t}$ satisfies $[(7.12)]$ at $w$. Let $w^{*}$ be the fixed point of $h^{*}\left(J^{* \times}\right)$ with some other $h^{*}$ and $J^{*}$. Since $b$ and $U$ are independent of $h$ and $J$, $t$ satisfies (7.12) at $w^{*}$, whenever the functions of (5.3) as well as $(P \circ \varepsilon)^{-1}$ are holomorphic at $j\left(w^{*}\right)$. Now we can find a function $t^{*}$ which satisfies $[7.12)$ at $w^{*}$ and such that $\operatorname{det}\left(t^{*}\left(w^{*}\right)\right) \neq 0$. Both $t$ and $t^{*}$ satisfy (7.12) at the points in a dense subset $S$ of $\mathfrak{S}_{1}^{r}$. Put $\mathfrak{f}=t^{*-1}$. We see that the entries of $\mathfrak{f}$ belong to $A_{0}$ and take algebraic values on $S$, and hence belong to $\mathcal{A}_{0}(\overline{\boldsymbol{Q}})$. Since $\mathrm{t}$ is holomorphic at $w^{*}$ if and only if $\mathfrak{f}$ is holomorphic at $w^{*}$, we see that $t$ satisfies [7.12) at $w^{*}$ whenever $t$ is holomorphic at $w^{*}$. This completes the proof.

One can prove the analogues of the above theorems for the derivatives of the functions $t$ satisfying (7.10) and (7.12). Also, the field $\overline{\boldsymbol{Q}}$ can be replaced by smaller fields depending on the weight. We have not pursued these points to their full extent in order not to obscure the ideas and to keep the paper short. In fact, almost all of the results in this section can be generalized (at least) to the group

$$
\left\{\left.\alpha \in G L_{n}(B)\right|^{t} \alpha^{\iota} \alpha \in F\right\}
$$

acting on $\mathfrak{H}_{n}^{r}$, which was treated in [10] and [11]. It should be noted that $\left(5.9_{\mathrm{a}, \mathrm{b}}\right),(7.12)$, or their generalizations can be utilized for defining the arithmeticity of automorphic forms, especially in the case of compact quotient.

As a final remark, we give here a paraphrase of Proposition 2.1 in terms of modular forms. Let $f$ be a (meromorphic) Hilbert modular form of weight $k$, i. e., an element of $\mathcal{A}_{k}$ with $B=M_{2}(F)$, and let $q$ be an element of $F$ such that $q_{\nu}^{\theta_{\nu}}>0$ or $<0$ according as $\nu \leqq r$ or $>r$. Put

$$
u(z)=f\left(q^{\theta_{1}} z, \cdots, q^{\theta_{r}} z, q^{\theta_{r+1}} \bar{z}, \cdots, q^{\theta_{g}} \bar{z}\right) \quad\left(z \in \mathfrak{S}_{1}\right),
$$

and suppose $k_{1}=\cdots=k_{r}=-k_{r+1}=\cdots=-k_{g}=\lambda$. Let $v(z)$ be a (meromorphic) modular form of weight $\lambda(2 r-g)$ with respect to a congruence subgroup of $S L_{2}(\boldsymbol{Z})$. Observe that 


$$
(u / v)(\gamma(z))=(u / v)(z)[(c z+d) /(c \bar{z}+d)]^{\lambda(g-r)}
$$

for $\gamma=\left(\begin{array}{ll}* & * \\ c & d\end{array}\right)$ in a congruence subgroup of $S L_{2}(\boldsymbol{Z})$.

PROPOSITION 7.9. Let $z_{0}$ be a point of $\mathscr{H}_{1}$ which generates an imaginary quadratic field $K$, and suppose both $f$ and $v$ are $\overline{\boldsymbol{Q}}$-rational. Then $(u / v)\left(z_{0}\right) \in \overline{\boldsymbol{Q}}$.

PROOF. Put $L=F K$ and define a $C M$-type $\Psi=\sum_{\nu=1}^{g} \sigma_{\nu}$ so that $\sigma_{\nu}=\theta_{\nu}$ on $F, z_{0}{ }_{\nu}=z_{0}$ or $\bar{z}_{0}$ according as $\nu \leqq r$ or $>r$. Then

$$
u\left(z_{0}\right) \sim \prod_{\nu=1}^{r} p\left(\sigma_{\nu}, \Psi\right)^{\lambda} \cdot \prod_{\nu=r+1}^{g} p\left(\sigma_{\nu}, \Psi\right)^{-\lambda} \sim v\left(z_{0}\right)
$$

by Proposition 2.1.

\section{The non-vanishing of $H^{1}\left(\Gamma \backslash \mathfrak{D}_{r}, \boldsymbol{R}\right)$.}

Let $\subseteq$ be an irreducible bounded symmetric domain, and $\Gamma$ a fixed-pointfree discontinuous group of holomorphic automorphisms of $\sqrt{S}$ such that $\Gamma \backslash \mathfrak{S}$ is compact. It was shown by Matsushima [4] that the first cohomology group of $\Gamma \backslash \subseteq$ over $\boldsymbol{R}$ vanishes unless $\subseteq$ is isomorphic to

$$
\mathfrak{D}_{r}=\mathfrak{D}(r, 1)=\left\{\left.\left(z_{1}, \cdots, z_{r}\right) \in \boldsymbol{C}^{r}\left|\sum_{k=1}^{r}\right| z_{k}\right|^{2}<1\right\} .
$$

The purpose of this section is to show that the last condition is actually meaningful. In other words, for each $r$, we can produce an example of $\Gamma$ acting on $\mathfrak{D}_{r}$ such that there is a holomorphic closed 1-form on $\Gamma \backslash \mathfrak{D}_{r}$ whose cohomology class is not 0 . The paper [3] by Kazhdan and a forthcoming work [1, Ch. VIII $]$ by Wallach should be mentioned as previous investigations on this topic. Our result can be stated as

THEOREM 8.1. Let $K, T$, and $\tau_{1}, \cdots, \tau_{g}$ be as in $\S 4$. Define $G_{\boldsymbol{Q}}$ by (4.1) and a subgroup $\Gamma_{1}$ of $G_{Q}$ by (4.14) with $N=1$ and with an arbitrary lattice $\mathfrak{n}$ of $K_{m}^{1}$. Let $\left(r_{\nu}, s_{\nu}\right)$ be the signature of $-i T^{\tau_{\nu}}$. Suppose $s_{1}=1$ and $s_{2}=\cdots=s_{g}=0$ (so that $\left.\mathfrak{D}=\mathfrak{D}_{m-1}\right)$. Then there is a subgroup $\Gamma$ of $\Gamma_{1}$ of finite index such that $\Gamma \backslash D$ has a closed holomorphic 1-form whose cohomology class is not 0. Moreover, we can find $m-1$ such 1 -forms $\xi_{1}, \cdots, \xi_{m-1}$ so that $\xi_{1} \wedge \cdots \wedge \xi_{m-1} \neq 0$.

This applies to both compact and non-compact quotients. Notice that, under the assumption on $s_{\nu}$ in the theorem, $\Gamma \backslash \mathfrak{D}$ is compact if $g>1$; it is not compact if $g=1$ and $r_{1}>1$.

To prove our theorem, we introduce the classical theta function

$$
\begin{array}{r}
\theta(u, Z ; a, b)=\Sigma_{X \in \mathbf{z}^{n}} e\left((1 / 2) \cdot{ }^{t}(x+a) Z(x+a)+{ }^{t}(x+a)(u+b)\right) \\
\left(u \in \boldsymbol{C}^{n}, Z \in \mathfrak{F}_{n}, a \in \boldsymbol{R}^{n}, b \in \boldsymbol{R}^{n}\right),
\end{array}
$$

where $e(v)=e^{2 \pi i v}$. It is well known that, for every $\gamma=\left(\begin{array}{ll}* & * \\ C & D\end{array}\right) \in \Delta_{2}$, one has 


$$
\begin{aligned}
& \theta\left({ }^{t}(C Z+D)^{-1} u, \gamma(Z) ; a, b\right) \\
= & \lambda_{\gamma} e\left(\left({ }^{t} a b-{ }^{t} a^{\prime} b^{\prime}\right) / 2\right) \operatorname{det}(C Z+D)^{1 / 2} e\left((1 / 2) \cdot{ }^{t} u(C Z+D)^{-1} C u\right) \theta\left(u, Z ; a^{\prime}, b^{\prime}\right),
\end{aligned}
$$

where $\left({ }^{t} a^{\prime},{ }^{t} b^{\prime}\right)=\left({ }^{t} a,{ }^{t} b\right) \gamma$, and $\lambda_{\gamma}$ is a fourth root of unity depending only on $\gamma$. With fixed $a_{1}, \cdots, a_{n}$ in $\boldsymbol{Q}^{n}$, define an $M_{n}(\boldsymbol{C})$-valued function $V$ on $\mathfrak{H}_{n}$ by $V(Z)=\left(v_{j k}(Z)\right), \quad v_{j k}(Z)=\left(\partial \theta / \partial u_{j}\right)\left(0, Z ; a_{k}, 0\right)$.

We see from (8.2) that

$$
V(\gamma(Z))=\lambda_{\gamma} \operatorname{det}(C Z+D)^{1 / 2} \cdot(C Z+D) V(Z) \quad \text { for every } \gamma=\left(\begin{array}{ll}
* & * \\
C & D
\end{array}\right) \in \Delta_{N},
$$

where $N$ is a suitably large even positive integer depending on $a_{1}, \cdots, a_{n}$. Moreover, as shown in [13, Proposition 1.2], given a point $Z_{0}$ of $\mathfrak{H}_{n}$, we can choose $a_{1}, \cdots, a_{n}$ so that $\operatorname{det}\left(V\left(Z_{0}\right)\right) \neq 0$. Now define a function $V^{\varepsilon}$ on $\mathfrak{D}$ by

$$
V^{\S}(z)=\operatorname{det}\left(\omega_{2}(z)\right)^{-1 / 2} \cdot{ }^{t} \omega_{2}(z)^{-1} V(\varepsilon(z)) \quad(z \in \mathfrak{D})
$$

with any choice of $\operatorname{det}\left(\omega_{2}(z)\right)^{-1 / 2}$, where $\varepsilon$ and $\omega_{2}$ are as in $\S 4$. By (4.16), $\left(4.10_{\mathrm{a}, \mathrm{b}}\right)$, and (3.9), we have

$$
V^{\varepsilon}(\alpha(z))=t(\alpha) \Pi_{\nu}^{\prime} \operatorname{det}\left(\mu_{\nu}(\alpha, z)\right) \cdot \Lambda(\alpha, z) V^{\varepsilon}(z) \quad \text { for } \quad \alpha \in \Gamma_{N}
$$

with a fourth root of unity $t(\alpha)$, where $\Pi_{\nu}^{\prime}$ is the product over all $\nu$ such that $r_{\nu} s_{\nu} \neq 0$. Obviously $t$ is a character of $\Gamma_{N}$. Choose the $a_{j}$ so that $\operatorname{det}\left(V^{\varepsilon}\right)$ does not vanish identically on $\mathbb{D}$.

Now assume that $r_{1}=m-1, s_{1}=1$, and $s_{2}=\cdots=s_{g}=0$. Then $\mathfrak{D}=\mathfrak{D}_{m-1}$. If $R$ is the first $m-1$ rows of $V^{\varepsilon},(8.3)$ implies that

$$
R(\alpha(z))=t(\alpha) \mu_{1}(\alpha, z) \lambda_{1}(\alpha, z) R(z) \quad \text { for } \quad \alpha \in \Gamma_{N} .
$$

Take an arbitrary non-vanishing column of $R(z)$ and call it $q(z)$, and its components $q_{1}, \cdots, q_{m-1}$. Let $z_{1}, \cdots, z_{m-1}$ be the components of the variable point $z$ on $\mathfrak{D}$. Define a 1 -form $\xi$ on $\mathscr{D}$ by

$$
\xi=\sum_{k=1}^{m-1} q_{k}(z) d z_{k}={ }^{t} q(z) d z .
$$

From (8.4) and (3.7), we see that $\xi_{\circ} \alpha=t(\alpha) \xi$ for all $\alpha \in \Gamma_{N}$. Therefore if $\Gamma=\operatorname{Ker}(t)$, then $\left[\Gamma_{N}: \Gamma\right] \leqq 4$, and $\xi$ defines a non-vanishing holomorphic 1 -form on $\Gamma \backslash \mathfrak{D}$. Since $R$ has rank $m-1$, we can choose $m-1$ columns of $R(z)$ which produce 1 -forms $\xi_{1}, \cdots, \xi_{m-1}$ such that $\xi_{1} \wedge \cdots \wedge \xi_{m-1} \neq 0$.

If $\Gamma \backslash \mathfrak{D}$ is compact, $\xi$ is closed and the cohomology class of $\xi$ is automatically non-vanishing; our proof is completed in that case. In the noncompact case, a further reasoning is necessary. As mentioned above, if $\mathfrak{D}=\mathfrak{D}_{m-1}$, the non-compact quotient occurs only when $g=1$. We are naturally interested only in the case $m>2$. First, to show that $\xi$ is closed, we use the unbounded domain 


$$
\left.3=\left\{(w, z) \in \boldsymbol{C}^{m-2} \times \boldsymbol{C} \mid i{ }^{t} \bar{w} S w+\bar{z}-z\right)>0\right\},
$$

which is isomorphic to $\mathfrak{D}_{m-1}$ and was introduced in [14]. Here $S$ is a diagonal skew-hermitian element of $G L_{m-2}(K)$ determined by $T$. We defined in [14, $\S 3]$ an embedding $\varepsilon^{\prime}$ of $\mathfrak{Z}$ into $\mathscr{S}_{m}$ similar to $\varepsilon$; so we can define everything with respect to 3 and $\varepsilon^{\prime}$ instead of $\mathscr{D}$ and $\varepsilon$. The function on 3 corresponding to $V^{\varepsilon}$ is defined by

$$
X(w, z)={ }^{t} \chi(w, z)^{-1} V\left(\varepsilon^{\prime}(w, z)\right) \quad((w, z) \in 3)
$$

with $\chi$ of $[14,(3.12)]$ (cf. (3.19) and Proposition 3.2 of [14]). Then $q$ corresponds to the first $m-1$ elements of a suitable column of $X(w, z)$, which can be given as

$$
\left[\begin{array}{c}
x_{1} \\
\vdots \\
x_{m}
\end{array}\right]={ }^{t} \chi(w, z)^{-1}\left[\begin{array}{c}
y_{1} \circ \varepsilon^{\prime} \\
\vdots \\
y_{m} \circ \varepsilon^{\prime}
\end{array}\right], \quad y_{k}=\left(\partial \theta / \partial u_{k}\right)(0, Z ; a, 0)
$$

with a suitable $a \in \boldsymbol{Q}^{m}$. Thus $\xi$ corresponds to a 1-form $\eta=\sum_{k=1}^{m-2} x_{k} d w_{k}+x_{m-1} d z$ on 3. Using the explicit form of $\chi$ in $[14,(3.12)]$, we find

$$
\begin{aligned}
& x_{k}=y_{k} \circ \varepsilon^{\prime}-\left(s_{k} w_{k} / 2\right) y_{m-1} \circ \varepsilon^{\prime}-\left(\delta s_{k} w_{k} / 2\right) y_{m} \circ \varepsilon^{\prime} \quad \text { if } k<m-1, \\
& x_{m-1}=\left(y_{m-1} \circ \varepsilon^{\prime}-\delta y_{m} \circ \varepsilon^{\prime}\right) / 2,
\end{aligned}
$$

where $s_{k}$ is the $k$-th diagonal element of $S$ and $\delta$ is a generator of $K$ used in $[14, \S 3]$. Now $[14,(3.7)]$ shows that

$$
d \varepsilon^{\prime}(w, z)=\left(\begin{array}{ccc}
0 & d w & \delta d w \\
{ }^{t} d w & d z-{ }^{t} w S d w & -\delta \cdot{ }^{t} w S d w \\
\delta \cdot{ }^{t} d w & -\delta \cdot{ }^{t} w S d w & -\delta^{2}\left(d z+{ }^{t} w S d w\right)
\end{array}\right) .
$$

Combining this with the well known relation

$$
\left(\partial^{2} \theta / \partial u_{j} \partial u_{k}\right)(u, Z ; a, b)=2 \pi i\left(1+\delta_{j k}\right)\left(\partial \theta / \partial Z_{j k}\right)(u, Z ; a, b),
$$

we can show by a direct calculation that $\partial x_{m-1} / \partial w_{k}=\partial x_{k} / \partial z$ and $\partial x_{j} / \partial w_{k}=$ $\partial x_{k} / \partial w_{j}$ if $j<m-1$ and $k<m-1$, which proves the desired closedness.

To show the non-vanishing of the cohomology-class, we go back to $D$ and take $T$ in the diagonal form $T=\operatorname{diag}\left[\zeta_{1}, \cdots, \zeta_{m}\right]$. Since the equivalence class of $T$ can be determined by its signature and the class of $\operatorname{det}(T)$ modulo $N_{K / Q}\left(K^{\times}\right)$, we can choose $\zeta_{1}, \cdots, \zeta_{m}$ so that $\zeta_{m-1} \zeta_{m}>0$ and $\zeta_{m-1} \zeta_{m} \notin N_{K / Q}\left(K^{\times}\right)$. (We are naturally assuming $m>2$.) Put

$$
G_{\boldsymbol{Q}}^{\prime}=\left\{\alpha \in G L_{2}(K) \mid \alpha T^{\prime} \cdot{ }^{t} \alpha=T^{\prime}\right\}, \quad T^{\prime}=\operatorname{diag}\left[\zeta_{m-1}, \zeta_{m}\right] .
$$

Then $G_{\boldsymbol{Q}}^{\prime}$ acts on the $\operatorname{disc} \mathfrak{D}_{1}$. Moreover, $\alpha \mapsto \operatorname{diag}\left[1_{m-1}, \alpha\right]$ gives an injection of $G_{Q}^{\prime}$ into $G_{Q}$, which is compatible with the embedding $z \mapsto\left(\begin{array}{l}0 \\ z\end{array}\right)$ of $\mathscr{D}_{1}$ into 
$\mathfrak{D}_{m-1}$. Take a congruence subgroup $\Gamma^{\prime}$ of $G_{Q}^{\prime}$ so that $\Gamma^{\prime}$ is mapped into $\Gamma_{N}$. Then $\Gamma^{\prime}$ has a subgroup of finite index $\Gamma^{\prime \prime}$ which is mapped into $\Gamma$. We can construct the above $\xi$ of (8.5) so that its pull-back to $\mathscr{D}_{1}$, say $\xi^{\prime}$, is not 0 . Our choice of $T^{\prime}$ implies that $\Gamma^{\prime \prime} \backslash \mathfrak{D}_{1}$ is compact, so that there is an element $\gamma$ of $\Gamma^{\prime \prime}$ and a point $w$ of $\mathfrak{D}_{1}$ such that $\int_{w}^{\gamma(w)} \xi^{\prime} \neq 0$. This shows the non-vanishing of the cohomology-class of $\xi$ on $\Gamma \backslash \mathfrak{D}$, and completes the proof.

Instead of taking the first $m-1$ columns, we can consider the $m$ columns $W_{\nu}$ of $V^{\varepsilon}$ corresponding to the $\nu$-th diagonal block $\Lambda_{\nu}(\alpha, z)$ of $\Lambda(\alpha, z)$ for $\nu>1$, when $g>1$. Then

$$
W_{\nu}(\alpha(z))=t(\alpha) \mu_{1}(\alpha, z) \Lambda_{\nu}(\alpha) W_{\nu}(z) \quad \text { for } \quad \alpha \in \Gamma_{N},
$$

where $\Lambda_{\nu}(\alpha)=\left(Q_{\nu}^{-1} \alpha^{\tau} \nu^{\rho} Q_{\nu}\right)^{\rho}$. Obviously $\Lambda_{\nu}$ is an injection of $G_{Q}$ into $U(m)$. Thus $W_{\nu}$ gives a rather interesting type of automorphic form.

\section{References}

[1] A. Borel and N. Wallach, Continuous cohomology, discrete subgroups and representations of reductive groups, to appear in Annals of Math. studies.

[2] B. Gross, On the periods of abelian integrals and a formula of Chowla and Selberg, Invent. Math. 45 (1978), 193-211.

[3] Kazhdan, Some applications of the Weil representation, preprint, 1976.

[4] Y. Matsushima, On the first Betti number of compact quotient spaces of higher dimensional symmetric spaces, Ann. of Math., 75 (1962), 312-330.

[5] K. Miyake, Models of certain automorphic function fields, Acta Math., 126 (1971), $245-307$.

[6] A. Selberg and S. Chowla, On Epstein's zeta-function, J. reine angew. Math., 227 (1967), 86-110.

[7] G. Shimura, On analytic families of polarized abelian varieties and automorphic functions, Ann. of Math., 78 (1963), 149-192.

[8] G. Shimura, On the field of definition for a field of automorphic functions, I, II, III, Ann. of Math., 80 (1964), 160-189; 81 (1965), 124-165; 83 (1966), 377-385.

[9] G. Shimura, Construction of class fields and zeta functions of algebraic curves, Ann. of Math., 85 (1967), 58-159.

[10] G. Shimura, Algebraic number fields and symplectic discontinuous groups, Ann. of Math., 86 (1967), 503-592.

[11] G. Shimura, On canonical models of arithmetic quotients of bounded symmetric domains, I, II, Ann. of Math., 91 (1970) 144-222; 92 (1970), 528-549.

[12] G. Shimura, On some arithmetic properties of modular forms of one and several variables, Ann. of Math., 102 (1975), 491-515.

[13] G. Shimura, On the derivatives of theta functions and modular forms, Duke Math. J., 44 (1977), 365-387.

[14] G. Shimura, The arithmetic of automorphic forms with respect to a unitary group, Ann. of Math., 107 (1978), 569-605.

[15] G. Shimura and Y. Taniyama, Complex multiplication of abelian varieties and its applications to number theory, Publ. Math. Soc. Japan, No. 6, 1961. 
[16] A. Weil, Sur les périodes aes intégrales abéliennes, Comm. Pure Appl. Math., 29 (1976), 813-819.

[17] T. Kubota, On the field extension by complex multiplication, Trans. Amer. Math. Soc., 118 (1965), 113-122.

\section{Goro SHIMURA}

Department of Mathematics

Princeton University

Princeton, New Jersey 08540

U.S.A. 